\title{
Theory of Light Scattering by an Isotropic System Composed of Anisotropic Units with Application to the Porod-Kratky Chain*
}

\author{
Kazuo NAGAI \\ Government Industrial Research Institute, Osaka, \\ Midorigaoka 1, Ikeda, Osaka, Japan.
}

(Received July 16, 1971)

\begin{abstract}
A theory of elastic (Rayleigh-Debye) light scattering by an isotropic system composed of anisotropic units is developed. Each unit is regarded optically as a point scatterer with three different principal polarizabilities. No assumption is introduced about the radial and orientational distributions for any pair of units. The only assumption is the random orientation of the system as a whole with respect to the light-scattering framework ("isotropic" system). Theory is formally adapted to infinitely dilute solutions of polymers of completely general structure. Detailed calculations are carried out for the Porod-Kratky wormlike chain. A method is suggested for determining the parameters of the Porod-Kratky chain through comparison of the theory with experimental data on polymer chains of moderate length. Chain-length dependences of various terms in expressions for reduced intensities are inferred for general chains from those for the Porod-Kratky chain. The correspondence of the Porod-Kratky chain with general chains is thereby discussed. A detailed comparison is also made of our results for the Porod-Kratky chain and general chains with those for the random chain reported by Utiyama and Kurata.

KEY WORDS Light Scattering / Isotropic System / Anisotropic Unit / Dilute Polymer Solution / Isotropic Inhomogeneous Solid / Porod-Kratky Chain / Random Chain /
\end{abstract}

As is well known Debye ${ }^{1}$ was the first to recognize the potentiality of light-scattering methods as a means to determine the molecular weight and the average spatial dimension of polymer chains in solution and developed a theory for linear chains. The model used by him is the so-called Gaussian chain; many identical units are connected by springs of zero rest length and of equal strength. Each unit is regarded optically as an isotropic point scatterer. Real chains depart from the Gaussian chain in many respects. Distribution functions of interunit distances deviate from Gaussian distributions by the discrete nature of real chains, i.e., fixed bond lengths and angles and hindered, internal rotations as well as by the excluded-volume effect. Units of a real chain are usually optical-

* Presented at U.S.-Japan Seminar on Statistical Mechanics and Spectroscopy of Polymers, University of Massachusetts, Amherst, Mass., U.S.A., August 2-6, 1971. ly anisotropic, no matter how units are defined; bonds, structural units, or Kuhn's segments. Numerous treatments have been published which are directed to refinement of Debye's theory.

In this paper we are concerned with the effect of the optical anisotropy of units. An important contribution toward this direction was already made by Utiyama and Kurata, ${ }^{2}$ who developed a theory for the random chain of optically anisotropic random links and found some important results (see later). We first develop a theory of elastic (Rayleigh-Debye) light scattering by a completely general model, i.e., an isotropic system composed of anisotropic units. Each unit is regarded most generally as a point scatterer with three different principal polarizabilities. No assumption is introduced about the radial and orientation distributions for any pair of units. The only assumption is the random orientation of the system as a whole with respect to the light-scattering framework ("isotropic" 


\section{K. NAGAI}

system), an assumption valid to polymer chains in solution unperturbed by external stress of any kind. The theory is then applied to general polymer chains and the Porod-Kratky wormlike chain. The similarity and disparity of results for the random chain ${ }^{2}$ and general chains and the Porod-Kratky chain are fully discussed.

Light scattering by polymer chains in solution has much in common with light scattering by an isotropic, inhomogeneous solid. ${ }^{3}$ Concerning the latter problem Goldstein and Michalik ${ }^{4}$ developed a very general theory, whose result might be usable for the present purpose. Unfortunately they introduced some simplifying assumptions: the axial symmetry of polarizability of each unit and an assumption about the orientational distribution between two units which together spoil complete generality. Stein and Wilson ${ }^{5}$ introduced further some simplifying assumptions and thereby obtained results that are much more tractable in the analysis of experimental results. The present theory avoids all these assumptions and hence some intermediate relations of this paper can be regarded as the most general solutions for the problem of light scattering by an isotropic, inhomogeneous solid.

\section{GENERAL THEORY}

Consider a monochromatic light beam which travels toward the positive $x$ axis of a laboratory coordinate system $x y z$ and is scattered at the origin by an isotropic system composed of anisotropic units (Figure 1). The incident beam may be unpolarized or vertically or horizontally polarized with respect to the scattering plane, i.e.,

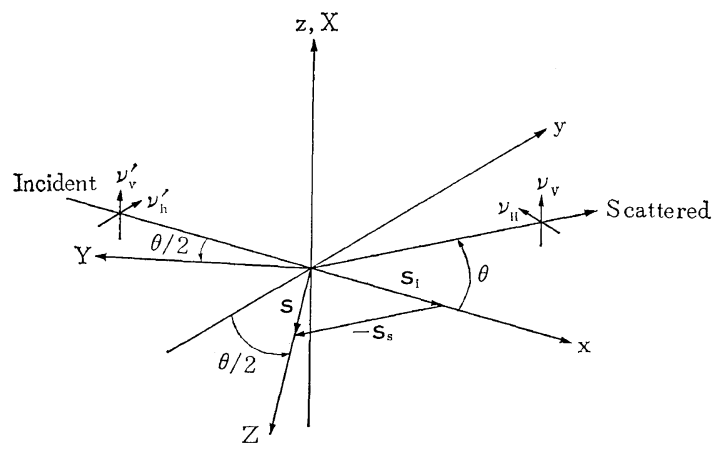

Figure 1. the $x y$ plane. Similarly, the unpolarized (total), or vertical or horizontal components of scattered light are measured in the scattering plane. ${ }^{*}$ The scattering system is composed of $n$ units, either identical or different, each of which is selected so small that it can be regarded as a point scatterer. Apart from a well-known factor to be multiplied (see, e.g., ref $2 \mathrm{a}$, eq 7 and 8 ) the intensity of scattered light is given by

$$
I=\sum_{i, j}\left\langle\left(\boldsymbol{\nu}^{T} \boldsymbol{\gamma}_{i} \boldsymbol{\nu}^{\prime}\right)\left(\boldsymbol{\nu}^{T} \boldsymbol{\gamma}_{j} \boldsymbol{\nu}^{\prime}\right) \exp \left(i k \mathbf{s} \cdot \mathbf{r}_{i j}\right)\right\rangle
$$

where $\nu^{\prime}$ and $\nu$ are the unit vectors along the electric vectors of incident and scattered beams; $\gamma_{i}$ and $\gamma_{j}$ are the polarizability tensors of units $i$ and $j$ (the excess polarizability tensors of units of a solute molecule in the case of solution); $i$ before $k$ is $(-1)^{1 / 2} ; k=2 \pi / \lambda$ with $\lambda$ the wave length of light in the medium; $\mathbf{s}=\mathbf{s}_{\mathbf{i}}-\mathbf{s}_{\mathbf{s}}$ with $\mathbf{s}_{\mathrm{i}}$ and $\mathbf{s}_{\mathrm{s}}$ being the unit vectors along the incident and scattered beams; $s=|\mathbf{s}|=2 \sin (\theta / 2)$ with $\theta$ the scattering angle; $\mathbf{r}_{i j}$ is the vector from unit $i$ to unit $j$; the superscript $T$ denotes the transpose of a vector; the summation on $i$ and $j$ is taken over all units; and the averaging is taken over all conformations of the system.

The averaging is carried out in two processes: (i) on the external coordinates, i.e., the free orientation of the system with a specified conformation with respect to the $x y z$ system and (ii) on the internal coordinates of the system.

$\boldsymbol{\gamma}_{i}$ can be expressed

$$
\gamma_{i}=\gamma_{i 1} \mu_{i 1} \mu_{i 1}^{T}+\gamma_{i 2} \mu_{i 2} \mu_{i 2}^{T}+\gamma_{i 3} \mu_{i 3} \mu_{i 3}^{T}
$$

where $\gamma_{i 1}, \gamma_{i 2}$, and $\gamma_{i 3}$ are the three principal values of the tensor $\boldsymbol{\gamma}_{i}$ and $\boldsymbol{\mu}_{i 1}, \boldsymbol{\mu}_{i 2}$, and $\boldsymbol{\mu}_{i 3}$ are the unit vectors along the corresponding axes. Substitution of $\boldsymbol{\gamma}_{i}$ and $\boldsymbol{\gamma}_{j}$, expressed as in eq 2 , into eq 1 yields $9 n^{2}$ terms, which can each be written representatively as

$$
I^{\prime}=\left\langle\gamma \gamma^{\prime}(\boldsymbol{\nu} \cdot \boldsymbol{\mu})\left(\boldsymbol{\nu} \cdot \boldsymbol{\mu}^{\prime}\right)\left(\boldsymbol{\nu}^{\prime} \cdot \boldsymbol{\mu}\right)\left(\boldsymbol{\nu}^{\prime} \cdot \boldsymbol{\mu}^{\prime}\right) \exp (i k \mathbf{s} \cdot \mathbf{r})\right\rangle
$$

* On the occasion of the U.S.-Japan Seminar Prof. W. Prins at Syracuse University kindly pointed out to the author the importance of the out-of-plane scattering, i.e., the scattering outside the $x y$ plane. This case appears to be able to treat within the framework of the present theory, i.e., by still leaving the scattered beam in plane while modifying $\nu$ and $\nu^{\prime}$ properly. We will discuss this problem in the near future. ${ }^{17}$ 
Separating the two processes of averaging we write

$$
\begin{aligned}
I^{\prime} & =\left\langle\gamma \gamma^{\prime}\langle\rangle_{\mathrm{ext}}\right\rangle_{\mathrm{int}}=\left\langle\gamma \gamma^{\prime} I^{\prime \prime}\right\rangle_{\mathrm{int}} \\
\boldsymbol{I}^{\prime \prime}= & \langle\rangle_{\operatorname{ext}}=\left(8 \pi^{2}\right)^{-1} \int(\boldsymbol{\nu} \cdot \boldsymbol{\mu})\left(\boldsymbol{\nu} \cdot \boldsymbol{\mu}^{\prime}\right)\left(\boldsymbol{\nu}^{\prime} \cdot \boldsymbol{\mu}\right)\left(\boldsymbol{\nu}^{\prime} \cdot \boldsymbol{\mu}^{\prime}\right) \\
& \times \exp (i k \mathbf{s} \cdot \mathbf{r}) \sin \theta^{\prime} \mathrm{d} \theta^{\prime} \mathrm{d} \varphi \mathrm{d} \psi
\end{aligned}
$$

where \langle\rangle$_{\text {ext }}$ and \langle\rangle$_{\text {int }}$ denote the averaging on the external and internal coordinates, i.e., processes (i) and (ii) above. For a specified (internal) conformation the relative spatial disposition of $\mu, \mu^{\prime}$, and $\mathbf{r}$ in space is definite, and the averaging on the external coordinates can be carried out by introduction of the Eulerian angles $\theta^{\prime} \varphi \psi$, as implied in eq 5 . The integral in eq 5 is of fundamental importance in theories of light scattering and is elementary in nature, but has never been evaluated (to our best knowledge) possibly because of its complexity.

To evaluate $I^{\prime \prime}$ in eq 5 we introduce two right-handed coordinate systems: one is the $X Y Z$ system fixed to the $x y z$ system and the other the $X^{\prime} Y^{\prime} Z^{\prime}$ system fixed to the scattering system. The $Z$ axis coincides with $\mathrm{s}$ and the $X$ axis with the $z$ axis (Figure 1). The $X Y Z$ and $x y z$ systems are correlated by

$$
\left[\begin{array}{l}
X \\
Y \\
Z
\end{array}\right]=\left[\begin{array}{ccc}
0 & 0 & 1 \\
-\cos (\theta / 2) & -\sin (\theta / 2) & 0 \\
\sin (\theta / 2) & -\cos (\theta / 2) & 0
\end{array}\right]\left[\begin{array}{l}
x \\
y \\
z
\end{array}\right]
$$

Let $\nu_{\mathrm{v}}{ }^{\prime}$ and $\nu_{\mathrm{h}}{ }^{\prime}$ stand for $\boldsymbol{\nu}^{\prime}$ for the vertically and horizontally polarized incident beams, and $\boldsymbol{\nu}_{\mathrm{V}}$ and $\boldsymbol{\nu}_{\mathrm{H}}$ for the vertically and horizontally polarized scattered beams. $\boldsymbol{\nu}_{\mathrm{v}}{ }^{\prime}, \boldsymbol{\nu}_{\mathrm{h}}{ }^{\prime}, \boldsymbol{\nu}_{\mathrm{V}}$, and $\boldsymbol{\nu}_{\mathrm{H}}$ are given in the $x y z$ system by $\left(\begin{array}{lll}0 & 0 & 1\end{array}\right)^{T},\left(\begin{array}{lll}0 & 1 & 0\end{array}\right)^{T}$, $\left(\begin{array}{lll}0 & 0 & 1\end{array}\right)^{T}$, and $(-\sin \theta \cos \theta \quad 0)^{T}$, and therefore by

$$
\boldsymbol{\nu}_{\mathrm{v}}{ }^{\prime}=\left[\begin{array}{l}
1 \\
0 \\
0
\end{array}\right], \quad \boldsymbol{\nu}_{\mathrm{h}}{ }^{\prime}=\left[\begin{array}{c}
0 \\
-\sin (\theta / 2) \\
-\cos (\theta / 2)
\end{array}\right], \quad \boldsymbol{\nu}_{\mathrm{v}}=\left[\begin{array}{l}
1 \\
0 \\
0
\end{array}\right],
$$

and

$$
\boldsymbol{\nu}_{\mathrm{H}}=\left[\begin{array}{c}
0 \\
\sin (\theta / 2) \\
-\cos (\theta / 2)
\end{array}\right], \quad \text { in the } X Y Z \text { system }
$$

The $Z^{\prime}$ axis is chosen to coincide with $\mathbf{r}$ and the $X^{\prime}$ and $Y^{\prime}$ axes are chosen arbitrarily (but, of course, so as to constiute a right-handed sys- tem), so that $\mathbf{r}, \boldsymbol{\mu}$, and $\mu^{\prime}$ are expressed

$$
\begin{array}{r}
\mathbf{r}=\left[\begin{array}{l}
0 \\
0 \\
r
\end{array}\right], \quad \mu=\left[\begin{array}{l}
\mu_{1} \\
\mu_{2} \\
\mu_{3}
\end{array}\right], \text { and } \mu^{\prime}=\left[\begin{array}{c}
\mu_{1}^{\prime} \\
\mu_{2}^{\prime} \\
\mu_{3}^{\prime}
\end{array}\right], \\
\text { in the } X^{\prime} Y^{\prime} Z^{\prime} \text { system }
\end{array}
$$

The $X^{\prime} Y^{\prime} Z^{\prime}$ system is correlated with the $X Y Z$ system by an orthogonal transformation matrix involving the Eulerian angles: $(X Y Z)^{T}=$

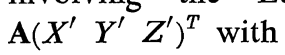

$$
\begin{aligned}
\mathbf{A}= & \mathbf{A}_{\varphi} \mathbf{A}_{\theta}, \mathbf{A}_{\psi} \\
= & {\left[\begin{array}{ccc}
\cos \varphi & -\sin \varphi & 0 \\
\sin \varphi & \cos \varphi & 0 \\
0 & 0 & 1
\end{array}\right]\left[\begin{array}{ccc}
\cos \theta^{\prime} & 0 & \sin \theta^{\prime} \\
0 & 1 & 0 \\
-\sin \theta^{\prime} & 0 & \cos \theta^{\prime}
\end{array}\right] } \\
& \times\left[\begin{array}{ccc}
\cos \psi & -\sin \psi & 0 \\
\sin \psi & \cos \psi & 0 \\
0 & 0 & 1
\end{array}\right]
\end{aligned}
$$

It is unnecessary to describe here the explicit geometrical meaning of the Eulerian angles except to say that the $Z$ and $Z^{\prime}$ axes are chosen as the polar axes, i.e., the angle between them being $\theta^{\prime}$.

With $\nu$ and $\nu^{\prime}$ expressed in the $X Y Z$ system (eq 7) while with $\mu$ and $\mu^{\prime}$ in the $X^{\prime} Y^{\prime} Z^{\prime}$ system (eq 8), $I^{\prime \prime}$ becomes

$$
\begin{aligned}
I^{\prime \prime}= & \left(8 \pi^{2}\right)^{-1} \int\left(\boldsymbol{\nu}^{T} \mathbf{A} \boldsymbol{\mu}\right)\left(\boldsymbol{\nu}^{\prime T} \mathbf{A} \boldsymbol{\mu}\right)\left(\boldsymbol{\nu}^{T} \mathbf{A} \boldsymbol{\mu}^{\prime}\right)\left(\boldsymbol{\nu}^{\prime T} \mathbf{A} \boldsymbol{\mu}^{\prime}\right) \\
& \times \exp \left(i k s r \cos \theta^{\prime}\right) \sin \theta^{\prime} \mathrm{d} \theta^{\prime} \mathrm{d} \varphi \mathrm{d} \psi
\end{aligned}
$$

The integral can in principle be evaluated by substituting $\mathbf{A}$ in eq 9 into 10, decomposing matrices, and performing the integration. This method is liable to lead to errors because of numerous terms ensuing. We have found a more systematic means of achieving this end.

Expressing the product of the two scalar factors as their direct product and rearranging by the direct-product theorem* we obtain ${ }^{6}$

* The direct product of the two matrices $\mathbf{a}=\left\{a_{i j}\right\}$ and $\mathbf{b}=\left\{b_{i j}\right\}$ is defined as

$$
\mathbf{a} \times \mathbf{b}=\left[\begin{array}{ccc}
\mathbf{a} b_{11} & \mathbf{a} b_{12} & \cdots \\
\mathbf{a} b_{21} & \mathbf{a} b_{22} & \cdots \\
& \cdots &
\end{array}\right]
$$

The important theorem of direct product needed below is

$$
\mathbf{a}_{1} \mathbf{a}_{2} \cdots \mathbf{a}_{k} \times \mathbf{b}_{1} \mathbf{b}_{2} \cdots \mathbf{b}_{k}=\left(\mathbf{a}_{1} \times \mathbf{b}_{1}\right)\left(\mathbf{a}_{2} \times \mathbf{b}_{2}\right) \cdots\left(\mathbf{a}_{k} \times \mathbf{b}_{k}\right)
$$




\section{K. NAGAI}

$$
\begin{array}{r}
\left(\boldsymbol{\nu}^{T} \mathbf{A} \boldsymbol{\mu}\right)\left(\boldsymbol{\nu}^{\prime T} \mathbf{A} \boldsymbol{\mu}\right)=\left(\boldsymbol{\nu}^{T} \mathbf{A}_{\varphi} \mathbf{A}_{\theta}, \mathbf{A}_{\psi} \boldsymbol{\mu}\right) \times\left(\boldsymbol{\nu}^{\prime T} \mathbf{A}_{\varphi} \mathbf{A}_{\theta}, \mathbf{A}_{\varphi} \boldsymbol{\mu}\right) \\
=\left(\boldsymbol{\nu}^{T} \times \boldsymbol{\nu}^{\prime T}\right)\left(\mathbf{A}_{\varphi} \times \mathbf{A}_{\varphi}\right)\left(\mathbf{A}_{\theta^{\prime}} \times \mathbf{A}_{\theta^{\prime}}\right)\left(\mathbf{A}_{\psi} \times \mathbf{A}_{\psi^{\prime}}\right)(\boldsymbol{\mu} \times \boldsymbol{\mu})
\end{array}
$$

where $x$ denotes the direct product of two matrices. ${ }^{*}$ The motivation for this procedure is to partition the same matrices into the same factors. ${ }^{6}$ The size of matrices in eq 11 can be reduced from $9 \times 9$ to $6 \times 6$ by utilizing the fact that these are written in the form of self-directproducts. Referring for the method elsewhere, ${ }^{6,7}$ we obtain

$$
\left(\boldsymbol{\nu}^{T} \mathbf{A} \boldsymbol{\mu}\right)\left(\boldsymbol{\nu}^{\prime T} \mathbf{A} \boldsymbol{\mu}\right)=\mathbf{y} \mathbf{B}_{\varphi} \mathbf{B}_{\theta}, \mathbf{B}_{\psi} \mathbf{X}
$$

with

$$
\begin{aligned}
& \mathbf{y}=\left(y_{1} y_{2} y_{3} y_{4} y_{5} y_{6}\right) \\
&=\left(\nu_{1} \nu^{\prime}, \nu_{1} \nu_{2}{ }^{\prime}+\nu_{2} \nu_{1}{ }^{\prime}, \nu_{1} \nu_{3}{ }^{\prime}+\nu_{3} \nu_{1}{ }^{\prime}, \nu_{2} \nu_{2}{ }^{\prime}, \nu_{2} \nu_{3}{ }^{\prime}\right. \\
&\left.+\nu_{3} \nu_{2}{ }^{\prime}, \nu_{3} \nu_{3}{ }^{\prime}\right) \\
& \mathbf{x}=\left(x_{1} x_{2} x_{3} x_{4} x_{5} x_{6}\right)^{T} \\
&=\left(\mu_{1}^{2}, \mu_{1} \mu_{2}, \mu_{1} \mu_{3}, \mu_{2}{ }^{2}, \mu_{2} \mu_{3}, \mu_{3}{ }^{2}\right)^{T} \\
& \mathbf{B}_{\varphi} \text { or } \mathbf{B}_{\psi}=\left[\begin{array}{cccccc}
\mathrm{c}^{2} & -2 \mathrm{cs} & 0 & \mathrm{~s}^{2} & 0 & 0 \\
\mathrm{cs} & \mathrm{c}^{2}-\mathrm{s}^{2} & 0 & -\mathrm{cs} & 0 & 0 \\
0 & 0 & \mathrm{c} & 0 & -\mathrm{s} & 0 \\
\mathrm{~s}^{2} & 2 \mathrm{cs} & 0 & \mathrm{c}^{2} & 0 & 0 \\
0 & 0 & \mathrm{~s} & 0 & \mathrm{c} & 0 \\
0 & 0 & 0 & 0 & 0 & 1
\end{array}\right]_{\varphi \text { or } \psi}
\end{aligned}
$$

$$
\mathbf{B}_{\theta^{\prime}}=\left[\begin{array}{cccccc}
\mathrm{c}^{2} & 0 & 2 \mathrm{cs} & 0 & 0 & \mathrm{~s}^{2} \\
0 & \mathrm{c} & 0 & 0 & \mathrm{~s} & 0 \\
-\mathrm{cs} & 0 & \mathrm{c}^{2}-\mathrm{s}^{2} & 0 & 0 & \mathrm{cs} \\
0 & 0 & 0 & 1 & 0 & 0 \\
0 & -\mathrm{s} & 0 & 0 & \mathrm{c} & 0 \\
\mathrm{~s}^{2} & 0 & -2 \mathrm{cs} & 0 & 0 & \mathrm{c}^{2}
\end{array}\right]_{\theta^{\prime}}
$$

where the subscript $\theta^{\prime}$ is implied to apply to c and $\mathrm{s}$ in the brackets as subscripts and $\mathrm{c}_{\theta^{\prime}}$ and $\mathrm{s}_{\theta^{\prime}}$ are the abbreviations of $\cos \theta^{\prime}$ and $\sin \theta^{\prime}$, and similarly with $\varphi$ and $\psi$. Similarly we obtain

$$
\begin{aligned}
& \left(\boldsymbol{\nu}^{T} \mathbf{A} \boldsymbol{\mu}^{\prime}\right)\left(\boldsymbol{\nu}^{\prime T} \mathbf{A} \boldsymbol{\mu}^{\prime}\right)=\mathbf{y} \mathbf{B}_{\varphi} \mathbf{B}_{\theta}, \mathbf{B}_{\phi} \mathbf{x}^{\prime}
\end{aligned}
$$

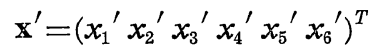

$$
\begin{aligned}
& =\left(\mu_{1}{ }^{\prime 2}, \mu_{1}{ }^{\prime} \mu_{2}{ }^{\prime}, \mu_{1}{ }^{\prime} \mu_{3}{ }^{\prime}, \mu_{2}{ }^{2}, \mu_{2}{ }^{\prime} \mu_{3}{ }^{\prime}, \mu_{3}{ }^{2}\right)^{T}
\end{aligned}
$$

Thus we have

$$
\begin{aligned}
& \left(\boldsymbol{\nu}^{T} \mathbf{A} \boldsymbol{\mu}\right)\left(\boldsymbol{\nu}^{\prime T} \mathbf{A} \boldsymbol{\mu}\right)\left(\boldsymbol{\nu}^{T} \mathbf{A} \boldsymbol{\mu}^{\prime}\right)\left(\boldsymbol{\nu}^{\prime T} \mathbf{A} \boldsymbol{\mu}^{\prime}\right) \\
& \quad=\left(\mathbf{y} \mathbf{B}_{\varphi} \mathbf{B}_{\theta} \cdot \mathbf{B}_{\psi} \mathbf{x}\right) \times\left(\mathbf{y} \mathbf{B}_{\varphi} \mathbf{B}_{\theta^{\prime}} \mathbf{B}_{\psi} \mathbf{x}^{\prime}\right) \\
& \quad=(\mathbf{y} \times \mathbf{y})\left(\mathbf{B}_{\varphi} \times \mathbf{B}_{\varphi}\right)\left(\mathbf{B}_{\theta^{\prime}} \times \mathbf{B}_{\theta^{\prime}}\right)\left(\mathbf{B}_{\psi} \times \mathbf{B}_{\psi}\right)(\mathbf{x} \times \mathbf{x})
\end{aligned}
$$

The size of matrices in eq 19 can similarly be reduced from $36 \times 36$ to $21 \times 21$ :

$$
\begin{aligned}
\left(\boldsymbol{\nu}^{T} \mathbf{A} \boldsymbol{\mu}\right)\left(\boldsymbol{\nu}^{\prime T} \mathbf{A} \boldsymbol{\mu}\right)\left(\boldsymbol{\nu}^{T} \mathbf{A} \boldsymbol{\mu}^{\prime}\right)\left(\boldsymbol{\nu}^{\prime T} \mathbf{A} \boldsymbol{\mu}^{\prime}\right) \\
=\left(y_{1}{ }^{2}, y_{1} y_{2}, \ldots, y_{6}{ }^{2}\right) \\
\quad \times \mathbf{D}_{\varphi} \mathbf{D}_{\theta^{\prime}} \mathbf{D}_{\varphi}\left(x_{1} x_{1}{ }^{\prime}, x_{1} x_{2}{ }^{\prime}+x_{2} x_{1}{ }^{\prime}, \ldots, x_{6} x_{6}{ }^{\prime}\right)^{T}(
\end{aligned}
$$

The matrices $\mathbf{D}_{\varphi}$ (or $\mathbf{D}_{\psi}$ ) and $\mathbf{D}_{\theta^{\prime}}$, are shown in Tables I and II, together with the row and column vectors, due to limitations of space. Upon substitution of eq 20 into eq 10 and integration on $\varphi$ and $\psi$, the 12 rows and columns out of 21 in $\mathbf{D}_{\varphi}$ and $\mathbf{D}_{\psi}$ become zero, as is apparent from Table I (the nonvanishing rows and columns are indicated by asterisk). Therefore, the corresponding rows and columns of $\mathbf{D}_{\theta^{\prime}}$ also become zero. Some of the nonvanishing rows and columns of $\mathbf{D}_{\varphi}$ and $\mathbf{D}_{\psi}$ are identical (of course after integration); those corresponding to $y_{1}{ }^{2}$ vs. $y_{4}{ }^{2}, y_{1} y_{6}$ vs. $y_{4} y_{6}$, and $y_{3}{ }^{2}$ vs. $y_{5}^{2}$, and similarly those corresponding to $x_{1} x_{1}{ }^{\prime}$ vs. $x_{4} x_{4}{ }^{\prime}, x_{6} x_{1}{ }^{\prime}+x_{1} x_{0}{ }^{\prime}$ vs. $x_{6} x_{4}{ }^{\prime}+x_{4} x_{6}{ }^{\prime}$, and $x_{3} x_{3}{ }^{\prime}$ vs. $x_{5} x_{5}^{\prime}$. This fact permits further reduction in the size of matrices. Thus deleting the vanishing rows and columns and then condensing the ensuing matrices we find

$$
\begin{aligned}
& I^{\prime \prime}=\frac{1}{128} \int_{0}^{\pi}\left[3 y_{1}{ }^{2}+2 y_{1} y_{4}+y_{2}{ }^{2}+3 y_{4}{ }^{2}, y_{1}{ }^{2}+6 y_{1} y_{4}-y_{2}{ }^{2}+y_{4}{ }^{2}, 4\left(y_{1}+y_{4}\right) y_{6}\right. \text {, } \\
& \left.4\left(y_{1}^{2}-2 y_{1} y_{4}+y_{2}^{2}+y_{4}^{2}\right), 4\left(y_{3}^{2}+y_{5}^{2}\right), 8 y_{6}^{2}\right] \\
& \times\left[\begin{array}{cccccc}
1+\mathrm{c}^{4} & 0 & \mathrm{c}^{2} \mathrm{~s}^{2} & 0 & 4 \mathrm{c}^{2} \mathrm{~s}^{2} & \mathrm{~s}^{4} \\
0 & \mathrm{c}^{2} & \mathrm{~s}^{2} & 0 & 0 & 0 \\
2 \mathrm{c}^{2} \mathrm{~s}^{2} & \mathrm{~s}^{2} & \mathrm{c}^{4}+\mathrm{s}^{4}+\mathrm{c}^{2} & 0 & -8 \mathrm{c}^{2} \mathrm{~s}^{2} & 2 \mathrm{c}^{2} \mathrm{~s}^{2} \\
0 & 0 & 0 & \mathrm{c}^{2} & \mathrm{~s}^{2} & 0 \\
\mathrm{c}^{2} \mathrm{~s}^{2} & 0 & -\mathrm{c}^{2} \mathrm{~s}^{2} & \mathrm{~s}^{2} & \left(\mathrm{c}^{2}-\mathrm{s}^{2}\right)^{2}+\mathrm{c}^{2} & \mathrm{c}^{2} \mathrm{~s}^{2} \\
\mathrm{~s}^{4} & 0 & \mathrm{c}^{2} \mathrm{~s}^{2} & 0 & 4 \mathrm{c}^{2} \mathrm{~s}^{2} & \mathrm{c}^{4}
\end{array}\right]_{\theta^{\prime}}\left[\begin{array}{c}
3 x_{1} x_{1}{ }^{\prime}+x_{4} x_{1}{ }^{\prime}+x_{1} x_{4}{ }^{\prime}+4 x_{2} x_{2}{ }^{\prime}+3 x_{4} x_{4}{ }^{\prime} \\
2 x_{1} x_{1}{ }^{\prime}+6\left(x_{4} x_{1}{ }^{\prime}+x_{1} x_{4}{ }^{\prime}\right)-8 x_{2} x_{2}{ }^{\prime}+2 x_{4} x_{4}{ }^{\prime} \\
4\left(x_{6} x_{1}{ }^{\prime}+x_{1} x_{6}{ }^{\prime}+x_{6} x_{4}{ }^{\prime}+x_{4} x_{6}{ }^{\prime}\right) \\
x_{1} x_{1}^{\prime}-x_{4} x_{1}^{\prime}-x_{1} x_{4}{ }^{\prime}+4 x_{2} x_{2}{ }^{\prime}+x_{4} x_{4}{ }^{\prime} \\
4\left(x_{3} x_{3}^{\prime}+x_{5} x_{5}^{\prime}\right) \\
8 x_{6} x_{6}^{\prime}
\end{array}\right] \\
& \times \exp \left(i k s r \cos \theta^{\prime}\right) \sin \theta^{\prime} \mathrm{d} \theta^{\prime}
\end{aligned}
$$


Going back from $\mathbf{y}$ to $\nu$ and from $\mathbf{x}$ to $\mu$ and grouping the same powers of $\cos \theta^{\prime}$ we obtain

$$
\begin{aligned}
I^{\prime \prime}= & (128)^{-1} \int_{0}^{\pi} \mathbf{V}\left(\mathbf{Q}_{0}+\mathbf{Q}_{1} \cos ^{2} \theta^{\prime}+\mathbf{Q}_{2} \cos ^{4} \theta^{\prime}\right) \mathbf{U} \\
& \times \exp \left(i k r s \cos \theta^{\prime}\right) \sin \theta^{\prime} \mathrm{d} \theta^{\prime}
\end{aligned}
$$

where

$$
\begin{aligned}
& \mathbf{V}=\left[1, \nu_{3}{ }^{2}+\nu_{3}{ }^{\prime 2}, \nu_{3}{ }^{2} \nu_{3}{ }^{2},\left(\boldsymbol{\nu} \cdot \boldsymbol{\nu}^{\prime}\right)^{2},\left(\boldsymbol{\nu} \cdot \boldsymbol{\nu}^{\prime}\right) \nu_{3} \nu_{3}{ }^{\prime}\right] \\
& \mathbf{U}=\left[1, \mu_{3}{ }^{2}+\mu_{3}{ }^{2}, \mu_{3}{ }^{2} \mu_{3}{ }^{\prime 2},\left(\boldsymbol{\mu} \cdot \boldsymbol{\mu}^{\prime}\right)^{2},\left(\boldsymbol{\mu} \cdot \boldsymbol{\mu}^{\prime}\right) \mu_{3} \mu_{3}{ }^{\prime}\right]^{T}
\end{aligned}
$$

$$
\mathbf{Q}_{0}=\left[\begin{array}{rrrrr}
1 & -5 & 3 & 2 & 12 \\
& 9 & -15 & 6 & -12 \\
& & 105 & 6 & -60 \\
& & & 4 & -8 \\
\text { sym } & & & & 48
\end{array}\right]
$$$$
\mathbf{Q}_{1}=\left[\begin{array}{rrrrr}
-10 & 18 & -30 & 12 & -24 \\
& -42 & 150 & -12 & -24 \\
& & -1050 & -60 & 600 \\
& & & -8 & 48 \\
\text { sym } & & & & -384
\end{array}\right]
$$

$$
\mathbf{Q}_{2}=\left[\begin{array}{rrrrr}
1 & -5 & 35 & 2 & -20 \\
& 25 & -175 & -10 & 100 \\
& & 1225 & 70 & -700 \\
& & & 4 & -40 \\
\text { sym } & & & & 400
\end{array}\right]
$$

Upon integration we have

$$
I^{\prime \prime}=(64)^{-1} \mathbf{V}\left(\mathbf{Q}_{0} F_{0}+\mathbf{Q}_{1} F_{1}+\mathbf{Q}_{2} F_{2}\right) \mathbf{U}
$$

where

$$
\begin{aligned}
F_{0} & =\frac{\sin z}{z}=\sum_{k=0}^{\infty}(-1)^{k} \frac{z^{2 k}}{(2 k+1) !} \\
F_{1} & =\left(\frac{1}{z}-\frac{2}{z^{3}}\right) \sin z+\frac{2 \cos z}{z^{2}} \\
& =\sum_{k=0}^{\infty}(-1)^{k} \frac{z^{2 k}}{(2 k) !(2 k+3)} \\
F_{2} & =\left(\frac{1}{z}-\frac{12}{z^{3}}+\frac{24}{z^{5}}\right) \sin z+\left(\frac{4}{z^{2}}-\frac{24}{z^{4}}\right) \cos z \\
& =\sum_{k=0}^{\infty}(-1)^{k} \frac{z^{2 k}}{(2 k) !(2 k+5)}
\end{aligned}
$$

with $z=k s r$. $I^{\prime \prime}$ is symmetric with respect to $\nu$ and $\nu^{\prime}$ and $\mu$ and $\mu^{\prime}$ as it should be from eq 10.

Polymer J., Vol. 3, No. 1, 1972
We proceed to substitute $I^{\prime \prime}$ into eq 4 and sum $I^{\prime}$ first on the respective three eigenvalues of the polarizabilities of units $i$ (unprimed) and $j$ (primed). Upon this summation, the first term unity in $\mathbf{U}$ leads to $\left(\gamma_{1}+\gamma_{2}+\gamma_{3}\right)\left(\gamma_{1}{ }^{\prime}+\gamma_{2}{ }^{\prime}+\gamma_{3}{ }^{\prime}\right)=$ $(\operatorname{Tr} \gamma)\left(\operatorname{Tr} \gamma^{\prime}\right)$, where $\operatorname{Tr}$ denotes the trace of a tensor; $\mu_{3}{ }^{2}+\mu_{3}{ }^{2}$ to $\left(\gamma_{1} \mu_{13}^{2}+\gamma_{2} \mu_{23}^{2}+\gamma_{3} \mu_{33}^{2}\right)\left(\gamma_{1}{ }^{\prime}+\gamma_{2}{ }^{\prime}\right.$ $\left.+\gamma_{3}{ }^{\prime}\right)+$ a similar term $=\boldsymbol{r}^{-2}\left[\left(\mathbf{r}^{T} \boldsymbol{\gamma} \mathbf{r}\right)\left(\operatorname{Tr} \boldsymbol{\gamma}^{\prime}\right)+\left(\mathbf{r}^{T} \boldsymbol{\gamma}^{\prime} \mathbf{r}\right)\right.$ $(\operatorname{Tr} \gamma)] ; \mu_{3}{ }^{2} \mu_{3}{ }^{2}$ to $\boldsymbol{r}^{-4}\left(\mathbf{r}^{T} \boldsymbol{\gamma} \mathbf{r}\right)\left(\mathbf{r}^{T} \boldsymbol{\gamma}^{\prime} \mathbf{r}\right) ; \quad\left(\boldsymbol{\mu} \cdot \boldsymbol{\mu}^{\prime}\right)^{2}$ to $\sum \gamma_{k} \gamma_{l}{ }^{\prime}\left(\boldsymbol{\mu}_{k} \cdot \boldsymbol{\mu}_{l}^{\prime}\right)^{2}=\operatorname{Tr} \boldsymbol{\gamma} \boldsymbol{\gamma}^{\prime} ;$ and $\left(\boldsymbol{\mu} \cdot \boldsymbol{\mu}^{\prime}\right) \mu_{3} \mu_{3}^{\prime}$ to $\sum \gamma_{k} \gamma_{l}{ }^{\prime}\left(\mu_{k} \cdot \mu_{l}{ }^{\prime}\right) \mu_{k 3} \mu_{l 3}^{\prime}=r^{-2} \mathbf{r}^{T} \gamma \gamma^{\prime} \mathbf{r}$. Some of these relations were established with the $X^{\prime} Y^{\prime} Z^{\prime}$ system, but they are invariant to a coordinate transformation and hence valid in any coordinate system. Thus we reach

$$
I=(64)^{-1} \sum_{i, j}\left\langle\left[\mathbf{V}\left(\mathbf{Q}_{0} F_{0}+\mathbf{Q}_{1} F_{1}+\mathbf{Q}_{2} F_{2}\right) \mathbf{U}^{\prime}\right]_{i j}\right\rangle
$$

where

$$
\mathbf{U}^{\prime}=\left[\begin{array}{c}
(\operatorname{Tr} \boldsymbol{\gamma})\left(\operatorname{Tr} \boldsymbol{\gamma}^{\prime}\right) \\
\boldsymbol{r}^{-2}\left[(\operatorname{Tr} \boldsymbol{\gamma}) \mathbf{r}^{T} \boldsymbol{\gamma}^{\prime} \mathbf{r}+\left(\operatorname{Tr} \boldsymbol{\gamma}^{\prime}\right) \mathbf{r}^{T} \boldsymbol{\gamma} \mathbf{r}\right] \\
\boldsymbol{r}^{-4}\left(\mathbf{r}^{T} \boldsymbol{\gamma} \mathbf{r}\right)\left(\mathbf{r}^{T} \boldsymbol{\gamma}^{\prime} \mathbf{r}\right) \\
\operatorname{Tr} \boldsymbol{\gamma} \boldsymbol{\gamma}^{\prime} \\
r^{-2} \mathbf{r}^{T} \gamma \boldsymbol{\gamma}^{\prime} \mathbf{r}
\end{array}\right]
$$

The subscript $i j$ in eq 32 implies the following substitutions to be made: $\boldsymbol{\gamma}$ and $\boldsymbol{\gamma}^{\prime}$ in $\mathbf{U}^{\prime}$ to $\boldsymbol{\gamma}_{i}$, and $\boldsymbol{\gamma}_{j}$, respectively, $\mathbf{r}$ in $\mathbf{U}^{\prime}$ to $\mathbf{r}_{i j}$ and $\boldsymbol{r}$ in $\mathbf{U}^{\prime}$ and $F$ 's to $r_{i j}$. This notation, introduced to simplify expressions, will be used throughout this paper. The average in eq 32 refers to the intrasystem average; the subscript int is hereafter omitted for brevity.

V's for the four combinations of vertically and horizontally polarized incident and scattered beams are given, according to eq 7 and 23 , by

$$
\begin{aligned}
& \mathbf{V}_{\mathrm{V}_{\mathrm{V}}}=\left(\begin{array}{lllll}
1 & 0 & 0 & 1 & 0
\end{array}\right) \\
& \mathbf{V}_{\mathrm{Vh}}=\mathbf{V}_{\mathrm{Hv}}=\left[1, \frac{1}{2}(1+\cos \theta), 0,0,0\right] \\
& \mathbf{V}_{\mathrm{Hh}}=\left[1,1+\cos \theta, \frac{1}{4}(1+\cos \theta)^{2}, \cos ^{2} \theta\right. \text {, } \\
& \left.\frac{1}{2}(1+\cos \theta) \cos \theta\right]
\end{aligned}
$$

where the first, capital subscript refers to the polarization of scattered beam, and the second, small subscript to that of incident beam (the same holds for $I$ and $R$ below).

Substituting eq 34-36 into eq 32 and decomposing matrices we obtain 


\section{K. NAGAI}

$$
\begin{aligned}
& I_{\nabla \mathrm{v}}=(64)^{-1} \sum_{i, j}\left\langle\left[\left(3 F_{0}+2 F_{1}+3 F_{2}\right)\left[(\operatorname{Tr} \gamma)\left(\operatorname{Tr} \boldsymbol{\gamma}^{\prime}\right)+2 \operatorname{Tr} \gamma \boldsymbol{\gamma}^{\prime}\right]+\left(F_{0}+6 F_{1}-15 F_{2}\right)\left[(\operatorname{Tr} \boldsymbol{\gamma}) \mathbf{r}^{T} \boldsymbol{\gamma}^{\prime} \mathbf{r}\right.\right.\right. \\
& \left.\left.\left.+\left(\operatorname{Tr} \boldsymbol{\gamma}^{\prime}\right) \mathbf{r}^{T} \boldsymbol{\gamma} \mathbf{r}+4 \mathbf{r}^{T} \boldsymbol{\gamma} \boldsymbol{\gamma}^{\prime} \mathbf{r}\right] \boldsymbol{r}^{-2}+3\left(3 F_{0}-30 F_{1}+35 F_{2}\right)\left(\mathbf{r}^{T} \boldsymbol{\gamma} \mathbf{r}\right)\left(\mathbf{r}^{T} \boldsymbol{\gamma}^{\prime} \mathbf{r}\right) r^{-4}\right]_{i j}\right\rangle \\
& I_{\mathrm{Vh}}=I_{\mathrm{HV}}=(128)^{-1} \sum_{i, j}\left\langle\left[-\left(3 F_{0}+2 F_{1}+3 F_{2}\right)(\operatorname{Tr} \gamma)\left(\operatorname{Tr} \gamma^{\prime}\right)-\left(F_{0}+6 F_{1}-15 F_{2}\right)\right.\right. \\
& \times\left[(\operatorname{Tr} \gamma) \mathbf{r}^{T} \boldsymbol{\gamma}^{\prime} \mathbf{r}+\left(\operatorname{Tr} \boldsymbol{\gamma}^{\prime}\right) \mathbf{r}^{T} \boldsymbol{\gamma} \mathbf{r}\right] r^{-2}-3\left(3 F_{0}-30 F_{1}+35 F_{2}\right)\left(\mathbf{r}^{T} \boldsymbol{\gamma} \mathbf{r}\right)\left(\mathbf{r}^{T} \boldsymbol{\gamma}^{\prime} \mathbf{r}\right) \boldsymbol{r}^{-4} \\
& +2\left(5 F_{0}+6 F_{1}-3 F_{2}\right) \operatorname{Tr} \gamma \gamma^{\prime}+12\left(F_{0}-6 F_{1}+5 F_{2}\right)\left(\mathbf{r}^{T} \gamma \gamma^{\prime} \mathbf{r}\right) r^{-2} \\
& +\left\{-\left(5 F_{0}-18 F_{1}+5 F_{2}\right)(\operatorname{Tr} \gamma)\left(\operatorname{Tr} \gamma^{\prime}\right)+\left(9 F_{0}-42 F_{1}+25 F_{2}\right)\right. \\
& \times\left[(\operatorname{Tr} \boldsymbol{\gamma}) \mathbf{r}^{T} \boldsymbol{\gamma}^{\prime} \mathbf{r}+\left(\operatorname{Tr} \boldsymbol{\gamma}^{\prime}\right) \mathbf{r}^{T} \boldsymbol{\gamma} \mathbf{r}\right] \boldsymbol{r}^{-2}-5\left(3 F_{0}-30 F_{1}+35 F_{2}\right)\left(\mathbf{r}^{T} \boldsymbol{\gamma} \mathbf{r}\right)\left(\mathbf{r}^{T} \boldsymbol{\gamma}^{\prime} \mathbf{r}\right) r^{-4} \\
& \left.\left.\left.+2\left(3 F_{0}-6 F_{1}-5 F_{2}\right) \operatorname{Tr} \gamma \gamma^{\prime}-4\left(3 F_{0}+6 F_{1}-25 F_{2}\right)\left(\mathbf{r}^{T} \gamma \gamma^{\prime} \mathbf{r}\right) r^{-2}\right\} \cos \theta\right]_{i j}\right\rangle \\
& I_{\mathrm{Hh}}=(256)^{-1} \sum_{i, j}\left\langle\left[\left(-13 F_{0}+2 F_{1}+19 F_{2}\right)(\operatorname{Tr} \gamma)\left(\operatorname{Tr} \gamma^{\prime}\right)+\left(F_{0}+54 F_{1}-95 F_{2}\right)\right.\right. \\
& \times\left[(\operatorname{Tr} \gamma) \mathbf{r}^{T} \gamma^{\prime} \mathbf{r}+\left(\operatorname{Tr} \gamma^{\prime}\right) \mathbf{r}^{T} \boldsymbol{\gamma} \mathbf{r}\right] r^{-2}+19\left(3 F_{0}-30 F_{1}+35 F_{2}\right)\left(\mathbf{r}^{T} \boldsymbol{\gamma} \mathbf{r}\right)\left(\mathbf{r}^{T} \gamma^{\prime} \mathbf{r}\right) r^{-4} \\
& +2\left(19 F_{0}-30 F_{1}+19 F_{2}\right) \operatorname{Tr} \gamma \gamma^{\prime}-4\left(15 F_{0}-102 F_{1}+95 F_{2}\right)\left(\mathbf{r}^{T} \gamma \gamma^{\prime} \mathbf{r}\right) r^{-2} \\
& +\left\{2\left(5 F_{0}-18 F_{1}+5 F_{2}\right)(\operatorname{Tr} \gamma)\left(\operatorname{Tr} \gamma^{\prime}\right)-2\left(9 F_{0}-42 F_{1}+25 F_{2}\right)\right. \\
& \times\left[(\operatorname{Tr} \boldsymbol{\gamma}) \mathbf{r}^{T} \boldsymbol{\gamma}^{\prime} \mathbf{r}+\left(\operatorname{Tr} \boldsymbol{\gamma}^{\prime}\right) \mathbf{r}^{T} \boldsymbol{\gamma} \mathbf{r}\right] r^{-2}+10\left(3 F_{0}-30 F_{1}+35 F_{2}\right)\left(\mathbf{r}^{T} \boldsymbol{\gamma} \mathbf{r}\right)\left(\mathbf{r}^{T} \boldsymbol{\gamma}^{\prime} \mathbf{r}\right) r^{-4} \\
& \left.+4\left(5 F_{0}-18 F_{1}+5 F_{2}\right) \operatorname{Tr} \gamma \gamma^{\prime}-8\left(9 F_{0}-42 F_{1}+25 F_{2}\right)\left(\mathbf{r}^{T} \gamma \gamma^{\prime} \mathbf{r}\right) r^{-2}\right\} \cos \theta \\
& +\left\{\left(35 F_{0}-30 F_{1}+3 F_{2}\right)(\operatorname{Tr} \gamma)\left(\operatorname{Tr} \gamma^{\prime}\right)-3\left(5 F_{0}-18 F_{1}+5 F_{2}\right)\right. \\
& \times\left[(\operatorname{Tr} \boldsymbol{\gamma}) \mathbf{r}^{T} \boldsymbol{\gamma}^{\prime} \mathbf{r}+\left(\operatorname{Tr} \boldsymbol{\gamma}^{\prime}\right) \mathbf{r}^{T} \boldsymbol{\gamma} \mathbf{r}\right] r^{-2}+3\left(3 F_{0}-30 F_{1}+35 F_{2}\right)\left(\mathbf{r}^{T} \boldsymbol{\gamma} \mathbf{r}\right)\left(\mathbf{r}^{T} \boldsymbol{\gamma}^{\prime} \mathbf{r}\right) \boldsymbol{r}^{-4} \\
& \left.\left.\left.+2\left(3 F_{0}+2 F_{1}+3 F_{2}\right) \operatorname{Tr} \gamma \gamma^{\prime}+4\left(F_{0}+6 F_{1}-15 F_{2}\right)\left(\mathbf{r}^{T} \gamma \gamma^{\prime} \mathbf{r}\right) r^{-2}\right\} \cos ^{2} \theta\right]_{i j}\right\rangle
\end{aligned}
$$

The complexity of these expressions will be compensated in part by their exactness. For an isotropic, inhomogeneous solid in which the mutual spatial dispositions of all units are definite, the averaging is taken over all pairs of units, the summation over $i$ and $j$ being thereby avoided..$^{3-5}$

Consider a special case where every unit is optically isotropic, i.e., $\gamma_{i 1}=\gamma_{i 2}=\gamma_{i 3}$. In this case $\mathbf{r}^{T} \gamma \mathbf{r}$ reduces to $\frac{1}{3} r^{2} \operatorname{Tr} \boldsymbol{\gamma}$; $\operatorname{Tr} \boldsymbol{\gamma} \boldsymbol{\gamma}^{\prime}$ to $\frac{1}{3}(\operatorname{Tr} \boldsymbol{\gamma})\left(\operatorname{Tr} \boldsymbol{\gamma}^{\prime}\right) ; \mathbf{r}^{T} \boldsymbol{\gamma} \boldsymbol{\gamma}^{\prime} \mathbf{r}$ to $\frac{1}{9} r^{2}(\operatorname{Tr} \gamma)\left(\operatorname{Tr} \gamma^{\prime}\right)$. Hence $I_{\mathrm{VV}}$, etc., reduce to

$$
\begin{aligned}
& \left.I_{\mathrm{VV}} \text { (iso }\right)=9^{-1} \sum_{i, j}\left\langle\left[F_{0}(\operatorname{Tr} \gamma)\left(\operatorname{Tr} \gamma^{\prime}\right)\right]_{i j}\right\rangle \\
& \left.\left.I_{\mathrm{Vh}} \text { (iso }\right)=I_{\mathrm{Hv}} \text { (iso }\right)=0 \\
& \left.I_{\mathrm{Hh}} \text { (iso }\right)=9^{-1} \sum_{i, j}\left\langle\left[F_{0}(\operatorname{Tr} \gamma)\left(\operatorname{Tr} \boldsymbol{\gamma}^{\prime}\right)\right]_{i j}\right\rangle \cos ^{2} \theta
\end{aligned}
$$

These are Debye's results. ${ }^{1}$ For the case of an- isotropic units it is convenient to write

$$
\begin{aligned}
& I_{\mathrm{Vv}}=I_{\mathrm{Vv}}(\text { iso })+I_{\mathrm{Vv}} \text { (aniso) } \\
& I_{\mathrm{Vh}}=I_{\mathrm{Hv}}=I_{\mathrm{Vh}}(\text { aniso })=I_{\mathrm{Hv}} \text { (aniso) } \\
& I_{\mathrm{Hh}}=I_{\mathrm{Hh}}(\text { iso })+I_{\mathrm{Hh}}(\text { aniso })
\end{aligned}
$$

where $I$ (aniso) is the contribution from anisotropic parts of polarizability of units (anisotropic scattering) and $I$ (iso), given by eq $40-42$, is the isotropic counterpart (isotropic scattering). $I$ (iso) is the intensity which we would have if every unit were isotropic, having the mean polarizability $\bar{\gamma}_{i}=\frac{1}{3} \operatorname{Tr} \gamma_{i}$.

For most models of interest the averages in eq 37-39 are difficult to calculate, but often become amenable when $F$ 's are expanded in in power series in $z=k s r$. Substituting eq 29-31 into eq 32 we have

$$
I=2^{-1} \sum_{i, j} \sum_{k=0}^{\infty}\left\langle\left[(-1)^{k}[(2 k+1) !(2 k+3)(2 k+5)]^{-1}(k s r)^{2 k} \mathbf{V} \mathbf{W}_{k} \mathbf{U}^{\prime}\right]_{i j}\right\rangle
$$

with

$$
\mathbf{W}_{k}=\left[\begin{array}{crrcc}
-\left(k^{2}+3 k+1\right) & k(k+3) & k(k-1) & 2 k^{2}+6 k+3 & -4 k(k+2) \\
& -k(k+5) & -5 k(k-1) & -2 k(k+2) & 2 k(4 k+5) \\
& & 35 k(k-1) & 2 k(k-1) & -20 k(k-1) \\
\text { sym } & & & 1 & 4 k \\
& & & & 4 k(2 k-5)
\end{array}\right]
$$


Light Scattering by an Isotropic System Composed of Anisotropic Units

$I_{\mathrm{VV}}$, etc., in series form can be obtained by sub- ment in the usual manner ${ }^{8}$ stituting eq $34-36$ into eq 46 .

$$
\bar{\gamma}=\left(n_{0} c V / 2 \pi N\right)(\mathrm{d} n / \mathrm{d} c)=\left(n_{0} M / 2 \pi N_{\mathrm{A}}\right)(\mathrm{d} n / \mathrm{d} c)
$$

\section{GENERAL POLYMER CHAINS}

In this section we consider light scattering by a polymer solution which is assumed so dilute that interactions between polymer chains are negligible; that is, we consider substantially single-chain scattering.

Regarding

$$
\bar{\gamma}=\frac{1}{3} \operatorname{Tr} \gamma=\frac{1}{3} \sum_{i=1}^{n} \operatorname{Tr} \gamma_{i}
$$

as the excess polarizability of a polymer chain and correlating it with the refractive index increwe find for the reduced intensity $R$

$$
\begin{gathered}
R=K c M_{\bar{\gamma}}^{-2} I \\
K=\left(4 \pi^{2} n_{0}^{2} / \lambda^{4} N_{\mathrm{A}}\right)(\mathrm{d} n / \mathrm{d} c)^{2}
\end{gathered}
$$

where $N$ is the number of polymer molecules in the scattering volume $V ; c$ is the concentration in $\mathrm{g} / \mathrm{cc} ; M$ is the molecular weight of a polymer; $n_{0}$ is the refractive index of a solvent; $N_{\mathrm{A}}$ is the Avogadro number; and $\mathrm{d} n / \mathrm{d} c$ is the refractive index increment of a polymer. Separating the isotropic and anisotropic parts of scattering and expanding only the latter in a series we obtain

$$
\begin{aligned}
& \boldsymbol{R}_{V_{\mathrm{v}}}=K c M_{\bar{\gamma}}^{-2}\left[f_{2}-f_{3}(s k)^{2}+\cdots+\sum_{i, j}\left\langle\left[\bar{\gamma} \bar{\gamma}^{\prime} F_{0}\right]_{i j}\right\rangle\right] \\
& R_{\mathrm{Vh}}=R_{\mathrm{Hv}}=K c M_{\bar{\gamma}}^{-2}\left[\frac{3}{4} f_{2}-f_{4}(s k)^{2}-f_{5}(s k)^{2} \cos \theta+\cdots\right] \\
& \left.R_{\mathrm{Hh}}=K c M_{\bar{\gamma}}^{-2}\left\{\frac{3}{4} f_{2}-f_{6}(s k)^{2}-f_{7}(s k)^{2} \cos \theta+\cdots+\left[\frac{1}{4} f_{2}-f_{8}(s k)^{2}+\cdots+\sum_{i, j}\left\langle\left[\bar{\gamma} \bar{\gamma}^{\prime} F_{0}\right)\right]_{i j}\right\rangle\right] \cos ^{2} \theta\right\} \\
& f_{2}=\frac{2}{45} \sum_{i, j}\left\langle\left[3 \operatorname{Tr} \gamma \gamma^{\prime}-(\operatorname{Tr} \gamma)\left(\operatorname{Tr} \gamma^{\prime}\right)\right]_{i j}\right\rangle=\frac{2}{15} \sum_{i, j}\left\langle\left[\operatorname{Tr} \hat{\gamma} \hat{\gamma}^{\prime}\right]_{i j}\right\rangle=\frac{2}{45}\left\langle 3 \operatorname{Tr} \gamma^{2}-(\operatorname{Tr} \gamma)^{2}\right\rangle=\frac{2}{15}\left\langle\operatorname{Tr} \hat{\gamma}^{2}\right\rangle \\
& f_{3}=\frac{1}{105} \sum_{i<j}\left\langle\left[-\frac{8}{9} r^{2}(\operatorname{Tr} \boldsymbol{\gamma})\left(\operatorname{Tr} \boldsymbol{\gamma}^{\prime}\right)-(\operatorname{Tr} \boldsymbol{\gamma}) \mathbf{r}^{T} \boldsymbol{\gamma}^{\prime} \mathbf{r}-\left(\operatorname{Tr} \boldsymbol{\gamma}^{\prime}\right) \mathbf{r}^{T} \boldsymbol{\gamma} \mathbf{r}+6 r^{2} \operatorname{Tr} \boldsymbol{\gamma} \boldsymbol{\gamma}^{\prime}-4 \mathbf{r}^{T} \boldsymbol{\gamma} \boldsymbol{\gamma}^{\prime} \mathbf{r}\right]_{i j}\right\rangle \\
& =\frac{1}{105} \sum_{i<j}\left\langle\left[-7 \bar{\gamma}\left(\mathbf{r}^{T} \hat{\gamma}^{\prime} \mathbf{r}\right)-7 \bar{\gamma}^{\prime}\left(\mathbf{r}^{T} \hat{\gamma} \mathbf{r}\right)+6 r^{2} \operatorname{Tr} \hat{\gamma} \hat{\gamma}^{\prime}-4 \mathbf{r}^{T} \hat{\gamma} \hat{\gamma}^{\prime} \mathbf{r}\right]_{i j}\right\rangle \\
& f_{4}=\frac{1}{210} \sum_{i<j}\left\langle\left[-3 r^{2}(\operatorname{Tr} \boldsymbol{\gamma})\left(\operatorname{Tr} \boldsymbol{\gamma}^{\prime}\right)+(\operatorname{Tr} \boldsymbol{\gamma}) \mathbf{r}^{T} \boldsymbol{\gamma}^{\prime} \mathbf{r}+\left(\operatorname{Tr} \boldsymbol{\gamma}^{\prime}\right) \mathbf{r}^{T} \boldsymbol{\gamma} \mathbf{r}+8 \boldsymbol{r}^{2} \operatorname{Tr} \boldsymbol{\gamma} \boldsymbol{\gamma}^{\prime}-3 \mathbf{r}^{T} \boldsymbol{\gamma} \boldsymbol{\gamma}^{\prime} \mathbf{r}\right]_{i j}\right\rangle \\
& =\frac{1}{210} \sum_{i<j}\left\langle\left[8 r^{2} \operatorname{Tr} \hat{\gamma} \hat{\gamma}^{\prime}-3 \mathbf{r}^{T} \hat{\gamma} \hat{\gamma}^{\prime} \mathbf{r}\right]_{i j}\right\rangle \\
& f_{5}=\frac{1}{210} \sum_{i<j}\left\langle\left[2 r^{2}(\operatorname{Tr} \boldsymbol{\gamma})\left(\operatorname{Tr} \boldsymbol{\gamma}^{\prime}\right)-3(\operatorname{Tr} \boldsymbol{\gamma}) \mathbf{r}^{T} \boldsymbol{\gamma}^{\prime} \mathbf{r}-3\left(\operatorname{Tr} \boldsymbol{\gamma}^{\prime}\right) \mathbf{r}^{T} \boldsymbol{\gamma} \mathbf{r}-3 \boldsymbol{r}^{2} \operatorname{Tr} \boldsymbol{\gamma} \boldsymbol{\gamma}^{\prime}+9 \mathbf{r}^{T} \boldsymbol{\gamma} \boldsymbol{\gamma}^{\prime} \mathbf{r}\right]_{i j}\right\rangle \\
& =\frac{1}{210} \sum_{i<j}\left\langle\left[-3 r^{2} \operatorname{Tr} \hat{\gamma} \hat{\gamma}^{\prime}+9 \mathbf{r}^{T} \hat{\gamma} \hat{\gamma}^{\prime} \mathbf{r}\right]_{i j}\right\rangle \\
& f_{6}=\frac{1}{210} \sum_{i<j}\left\langle\left[-r^{2}(\operatorname{Tr} \gamma)\left(\operatorname{Tr} \boldsymbol{\gamma}^{\prime}\right)-2(\operatorname{Tr} \boldsymbol{\gamma}) \mathbf{r}^{T} \boldsymbol{\gamma}^{\prime} \mathbf{r}-2\left(\operatorname{Tr} \boldsymbol{\gamma}^{\prime}\right) \mathbf{r}^{T} \boldsymbol{\gamma} \mathbf{r}+5 r^{2} \operatorname{Tr} \gamma \boldsymbol{\gamma}^{\prime}+6 \mathbf{r}^{T} \gamma \gamma^{\prime} \mathbf{r}\right]_{i j}\right\rangle \\
& =\frac{1}{210} \sum_{i<j}\left\langle\left[5 r^{2} \operatorname{Tr} \hat{\gamma} \hat{\gamma}^{\prime}+6 \mathbf{r}^{T} \hat{\gamma} \hat{\gamma}^{\prime} \mathbf{r}\right]_{i j}\right\rangle \\
& f_{7}=\frac{1}{210} \sum_{i<j}\left\langle\left[-2 r^{2}(\operatorname{Tr} \boldsymbol{\gamma})\left(\operatorname{Tr} \boldsymbol{\gamma}^{\prime}\right)+3(\operatorname{Tr} \boldsymbol{\gamma}) \mathbf{r}^{T} \boldsymbol{\gamma}^{\prime} \mathbf{r}+3\left(\operatorname{Tr} \boldsymbol{\gamma}^{\prime}\right) \mathbf{r}^{T} \boldsymbol{\gamma} \mathbf{r}-4 \boldsymbol{r}^{2} \operatorname{Tr} \boldsymbol{\gamma} \boldsymbol{\gamma}^{\prime}+12 \mathbf{r}^{T} \boldsymbol{\gamma} \boldsymbol{\gamma}^{\prime} \mathbf{r}\right]_{i j}\right\rangle \\
& =\frac{1}{210} \sum_{i<j}\left\langle\left[21 \bar{\gamma}\left(\mathbf{r}^{T} \hat{\gamma}^{\prime} \mathbf{r}\right)+21 \bar{\gamma}^{\prime}\left(\mathbf{r}^{T} \hat{\gamma} \mathbf{r}\right)-4 \mathbf{r}^{2} \operatorname{Tr} \hat{\gamma} \hat{\gamma}^{\prime}+12 \mathbf{r}^{T} \hat{\gamma} \hat{\gamma}^{\prime} \mathbf{r}\right]_{i j}\right\rangle \\
& f_{8}=\frac{1}{210} \sum_{i<j}\left\langle\left[-\frac{25}{9} r^{2}(\operatorname{Tr} \boldsymbol{\gamma})\left(\operatorname{Tr} \boldsymbol{r}^{\prime}\right)+3(\operatorname{Tr} \boldsymbol{\gamma}) \mathbf{r}^{T} \boldsymbol{\gamma}^{\prime} \mathbf{r}+3\left(\operatorname{Tr} \boldsymbol{r}^{\prime}\right) \mathbf{r}^{T} \boldsymbol{\gamma} \mathbf{r}+3 \boldsymbol{r}^{2} \operatorname{Tr} \boldsymbol{\gamma} \boldsymbol{\gamma}^{\prime}-2 \mathbf{r}^{T} \boldsymbol{\gamma} \boldsymbol{\gamma}^{\prime} \mathbf{r}\right]_{i j}\right\rangle \\
& \left.=\frac{1}{210} \sum_{i<j}\left\langle\left[7 \bar{\gamma}^{\left(\mathbf{r}^{T}\right.} \hat{\gamma}^{\prime} \mathbf{r}\right)+7 \bar{\gamma}^{\prime}\left(\mathbf{r}^{T} \hat{\gamma} \mathbf{r}\right)+3 r^{2} \operatorname{Tr} \hat{\gamma} \hat{\gamma}^{\prime}-2 \mathbf{r}^{T} \hat{\gamma} \hat{\gamma}^{\prime} \mathbf{r}\right]_{i j}\right\rangle
\end{aligned}
$$




\section{K. NAGAI}

Here $\bar{\gamma}_{i}=\frac{1}{3} \operatorname{Tr} \gamma_{i}$ and $\hat{\gamma}$ is the traceless part of $\gamma$, i.e., $\hat{\gamma}_{i}=\gamma_{i}-\bar{\gamma}_{i} \mathbf{E}_{3}, \mathbf{E}_{3}$ being the unit matrix of order 3. The $f_{2}$ term, related to the optical anisotropy $\frac{3}{2}\left\langle\operatorname{Tr} \hat{\gamma}^{2}\right\rangle$, has been well known in connection with the depolarization ratio in the RayleighDebye $^{9}$ and Raman scattering. The other $f$ terms are new. For the random chain all the $f$ and higher [in $\left.(s k)^{2}\right]$ terms except for $f_{2}$ vanish.

All $f$ are calculable for any model of polymer chains, either hypothetical or realistic, under the assumption of the absence of the excluded volume. It seems impossible, however, to determine these $f$, except for $f_{2}$, from experimental data of $R$, the unknowns being too many compared with the observables. In this paper we calculate the $f$ for the Porod-Kratky chain. Methods of adjusting this model chain to a real chain are by no means unique, and hence so is the relation of the parameters of this model chain with structures of a real chain. ${ }^{10,11}$ However we expect this model chain to predict at least the correct chain-length dependences of $f$ for a real chain.

\section{POROD-KRATKY CHAIN}

The problem of light scattering by the PorodKratky chain ${ }^{12}$ composed of anisotropic units was formulated first by Hermans and Ullman. ${ }^{13}$ However, their theory was not carried through far enough to be usable for analysis of experimental data. An unpleasant assumption concerning the optical anisotropy of units was introduced, which will severely limit the applicability of the theory (see later). Methods explored by them for obtaining various averages are powerful and sufficiently general for the present purpose, and we will make full use of them in this paper.

Consider the Porod-Kratky chain of contour length $t$ and persistent length $a$. For its optical property we assume that the chain has, per unit length, the three principal polarizabilities, $\alpha_{1}$ along the contour and $\alpha_{2}$ along the two directions perpendicular to the contour, i.e., we assume the cylindrical symmetry $\alpha_{3}=\alpha_{2}$. A lower symmetry $\alpha_{3} \neq \alpha_{2}$ is not practical for this model. Units $i$ and $j$ are regarded as referring to the increments $\mathrm{d} i$ and $\mathrm{d} j$ of the chain, which depart by the contour lengths $i$ and $j$ from one end.

The polarizability tensor $\gamma_{i}$ of unit $i$ (but per unit length) can be expressed

$$
\begin{aligned}
\boldsymbol{\gamma}_{i} & =\left(\alpha_{1}-\alpha_{2}\right) \boldsymbol{\mu}_{i} \boldsymbol{\mu}_{i}{ }^{T}+\alpha_{2} \mathbf{E}_{3} \\
& =\Delta \alpha \boldsymbol{\mu}_{i} \boldsymbol{\mu}_{i}{ }^{T}+\left(\bar{\alpha}-\frac{1}{3} \Delta \alpha\right) \mathbf{E}_{3}
\end{aligned}
$$

where $\mu_{i}$ is the unit vector along the contour of unit $i ; \bar{\alpha}=\frac{1}{3}\left(\alpha_{1}+2 \alpha_{2}\right)$ and $\Delta \alpha=\alpha_{1}-\alpha_{2}$ are the mean and anisotropic polarizabilities per unit length of the chain. We immediately have

$$
\begin{aligned}
\mathbf{r}_{i j}{ }^{T} \gamma_{i} \mathbf{r}_{i j}= & \Delta \alpha\left(\mathbf{r}_{i j} \cdot \mu_{i}\right)^{2}+\left(\bar{\alpha}-\frac{1}{3} \Delta \alpha\right) r_{i j}^{2} \\
\operatorname{Tr} \gamma_{i} \gamma_{j}= & (\Delta \alpha)^{2}\left(\boldsymbol{\mu}_{i} \cdot \mu_{j}\right)^{2}+3 \bar{\alpha}^{2}-\frac{1}{3}(\Delta \alpha)^{2} \\
\mathbf{r}_{i j}{ }^{T} \gamma_{i} \gamma_{j} \mathbf{r}_{i j}= & (\Delta \alpha)^{2}\left(\mathbf{r}_{i j} \cdot \boldsymbol{\mu}_{i}\right)\left(\mathbf{r}_{i j} \cdot \boldsymbol{\mu}_{j}\right)\left(\boldsymbol{\mu}_{i} \cdot \boldsymbol{\mu}_{j}\right) \\
& +\Delta \alpha\left(\bar{\alpha}-\frac{1}{3} \Delta \alpha\right)\left[\left(\mathbf{r}_{i j} \cdot \boldsymbol{\mu}_{i}\right)^{2}+\left(\mathbf{r}_{i j} \cdot \boldsymbol{\mu}_{j}\right)^{2}\right] \\
& +\left(\bar{\alpha}-\frac{1}{3} \Delta \alpha\right)^{2} r_{i j}{ }^{2}
\end{aligned}
$$

From eq 55-61 and 63-65 it is clear that we need the following averages: $\left\langle r_{i j}{ }^{2}\right\rangle,\left\langle\left(\mu_{i} \cdot \mu_{j}\right)^{2}\right\rangle$, $\left\langle\left(\mathbf{r}_{i j} \cdot \boldsymbol{\mu}_{i}\right)^{2}\right\rangle$ [or equivalently $\left.\left\langle\left(\mathbf{r}_{i j} \cdot \boldsymbol{\mu}_{j}\right)^{2}\right\rangle\right],\left\langle\boldsymbol{r}_{i j}^{2}\left(\boldsymbol{\mu}_{i}\right.\right.$. $\left.\left.\boldsymbol{\mu}_{j}\right)^{2}\right\rangle$, and $\left\langle\left(\mathbf{r}_{i j} \cdot \boldsymbol{\mu}_{i}\right)\left(\mathbf{r}_{i j} \cdot \boldsymbol{\mu}_{j}\right)\left(\boldsymbol{\mu}_{i} \cdot \boldsymbol{\mu}_{j}\right)\right\rangle$. For the Porod-Kratky chain these averages do not depend on where on the chain the pair of units $i$ and $j$ is selected if $j-i$ is kept constant. Therefore it suffices to calculate these averages for the two terminal units, i.e., $i=0$ and $j=t$. We omit the subscript to $\mathbf{r}$, now $\mathbf{r}$ being the end-toend vector. All the required averages can be cast into

$$
u_{k l m n}=\langle\Psi\rangle=\left\langle r^{k}\left(\mathbf{r} \cdot \boldsymbol{\mu}_{0}\right)^{l}\left(\mathbf{r} \cdot \boldsymbol{\mu}_{t}\right)^{m}\left(\boldsymbol{\mu}_{0} \cdot \boldsymbol{\mu}_{t}\right)^{n}\right\rangle
$$

Hermans and Ullman ${ }^{13}$ developed a method for calculating averages like eq 66 for the PorodKratky chain in the absence of the excludedvolume effect. They treated simpler averages of the form $\left\langle\boldsymbol{r}^{k}\left(\mathbf{r} \cdot \boldsymbol{\mu}_{t}\right)^{m}\right\rangle$ a special case of eq 66 , but their method is applicable to more complex averages in eq 66. We follow their method exactly.

The first step of Hermans and Ulllman's theory is to derive a differential equation for a distribution function $f\left(\mathbf{r}, \mu_{t}, t\right)$ for one end (with $t=t$ ) when the other end (with $t=0$ ) is at the origin of a cartesian coordinate system $x y z$ and has the initial tangent $\mu_{0}$. The derived differential equation is insoluble exactly, but the required averages can still be obtained by utilizing it in the following manner. The differential equation for $f\left(\mathbf{r}, \boldsymbol{\ell}_{t}, t\right)$ is converted to that for its dimensionless Laplace transform with respect to $t$ :

$$
\begin{aligned}
f^{\prime}=f^{\prime}\left(\mathbf{r}, \mu_{t}, p\right) & =\mathrm{L}_{\mathrm{d} 1}\left[f\left(\mathbf{r}, \mu_{t}, t\right)\right] \\
& =p \int_{0}^{\infty} f\left(\mathbf{r}, \mu_{t}, t\right) \mathrm{e}^{-t p} \mathrm{~d} t
\end{aligned}
$$


Light Scattering by an Isotropic System Composed of Anisotropic Units

that is,

$$
p f^{\prime}+\left(\mu_{t} \cdot \operatorname{grad}_{\mathbf{r}} f^{\prime}\right)=\lambda \nabla^{2} f^{\prime}
$$

where $\lambda=(2 a)^{-1}, \operatorname{grad}_{\mathrm{r}}=(\partial / \partial x, \partial / \partial y, \partial / \partial z)^{T}$, and

$$
\nabla^{2}=\frac{1}{\sin \theta} \frac{\partial}{\partial \theta}\left(\sin \theta \frac{\partial}{\partial \theta}\right)+\frac{1}{\sin ^{2} \theta} \frac{\partial^{2}}{\partial \varphi^{2}}
$$

with $\mu_{t}=(\cos \theta, \sin \theta \cos \varphi, \sin \theta \sin \varphi)^{T}$, (these $\theta$ and $\varphi$ should not be confused with those defined previously, i.e., the scattering angle and one of the Eulerian angles; similarly $\lambda$ in eq 68 should not be confused with the wave length of light). It is to be noted that the dimensionless Laplace transform defined in eq 67 differs from the ordinary Laplace transform

$$
\begin{aligned}
\mathrm{L}_{\mathrm{od}}\left[f\left(\mathbf{r}, \mu_{t}, t\right)\right] & =\int_{0}^{\infty} f\left(\mathbf{r}, \mu_{t}, t\right) \mathrm{e}^{-t p} \mathrm{~d} t \\
& =p^{-1} f^{\prime}
\end{aligned}
$$

We find immediately

$$
f=\mathrm{L}_{\mathrm{d} 1}^{-1}\left[f^{\prime}\right]=\mathrm{L}_{\mathrm{od}}^{-1}\left[p^{-1} f^{\prime}\right]
$$

Multiplication of $\Psi$ in eq 66 to eq 68 and integration over the entire $\mathbf{r}, \mu_{t}$ space yield

$$
p\langle\Psi\rangle^{\prime}-p \Psi_{0}=\left\langle\boldsymbol{\mu}_{t} \cdot \operatorname{grad}_{\mathbf{r}} \Psi\right\rangle^{\prime}+\lambda\left\langle\nabla^{2} \Psi\right\rangle^{\prime}
$$

where $\Psi_{0}$ is the value of $\Psi$ at the origin and the primed averages refer to those in the $p$ space, i.e., \langle\rangle$^{\prime}=\mathrm{L}_{\mathrm{d} 1}\left[\langle>]\right.$. Similarly let $u_{k l m n}^{\prime}=$ $\mathrm{L}_{\mathrm{d} 1}\left[u_{k l m n}\right]$. Repetitive use of eq 71 with proper $\Psi$ leads, after some lengthy but straightforward calculations (see ref 13) to:

$$
\begin{aligned}
& (p+2 \lambda) u_{0010}^{\prime}=1 \\
& p u_{2000}^{\prime}=2 u_{0010}^{\prime} \\
& (p+6 \lambda) u_{0002}^{\prime}=p+2 \lambda \\
& (p+2 \lambda) u_{0101}^{\prime}=u_{0002}^{\prime} \\
& (p+12 \lambda) u_{0012}^{\prime}=u_{0002}^{\prime}+2 \lambda u_{0010}^{\prime}+4 \lambda u_{0101}^{\prime} \\
& (p+6 \lambda) u_{2002}^{\prime}=2 u_{0012}^{\prime}+2 \lambda u_{2000}^{\prime} \\
& p u_{0200}^{\prime}=2 u_{0101}^{\prime} \\
& (p+6 \lambda) u_{0111}^{\prime}=u_{0101}^{\prime}+u_{0012}^{\prime}+2 \lambda u_{0200}^{\prime}
\end{aligned}
$$

Solving these relations we obtained

$$
\begin{aligned}
u_{2000}^{\prime}= & \left\langle r^{2}\right\rangle^{\prime}=\frac{1}{\lambda}\left(\frac{1}{p}-\frac{1}{p+2 \lambda}\right) \\
u_{0002}^{\prime}= & \left\langle\left(\mu_{0} \cdot \mu_{t}\right)^{2}\right\rangle^{\prime}=1-\frac{4 \lambda}{p+6 \lambda} \\
u_{0200}^{\prime}= & \left\langle\left(\mathbf{r} \cdot \mu_{0}\right)^{2}\right\rangle^{\prime}=\frac{1}{3 \lambda}\left(\frac{1}{p}-\frac{1}{p+6 \lambda}\right) \\
u_{2002}^{\prime}= & \left\langle r^{2}\left(\mu_{0} \cdot \mu_{t}\right)^{2}\right\rangle^{\prime} \\
= & \frac{1}{15 \lambda}\left(\frac{5}{p}-\frac{6}{p+2 \lambda}+\frac{5}{p+6 \lambda}-\frac{4}{p+12 \lambda}\right) \\
u_{0111}^{\prime}= & \left\langle\left(\mathbf{r} \cdot \mu_{0}\right)\left(\mathbf{r} \cdot \mu_{t}\right)\left(\mu_{0} \cdot \mu_{t}\right)\right\rangle^{\prime} \\
= & \frac{1}{180 \lambda}\left(\frac{20}{p}+\frac{9}{p+2 \lambda}-\frac{5}{p+6 \lambda}\right. \\
& \left.+\frac{60 \lambda}{(p+6 \lambda)^{2}}-\frac{24}{p+12 \lambda}\right)
\end{aligned}
$$

Laplace inversion, i.e., $\quad \mathrm{L}_{\mathrm{d} 1}^{-1}\left[u_{k l m n}^{\prime}\right]=\mathrm{L}_{\text {od }}^{-1}$ $\cdot\left[p^{-1} u_{k l m n}^{\prime}\right]$ yields the required $u_{k l m n}$. Inversion at this point is not wise. ${ }^{13}$ All $u$ are followed by the following integration

$$
I(t)=\iint_{i<j} u(j-i) \mathrm{d} i \mathrm{~d} j=\int_{0}^{t} i u(t-i) \mathrm{d} i
$$

Note that the above integral is of the so-called convolution type. Remembering that the ordinary Laplace transform of a convolution integral is the product of those of the constituent functions we find

$$
\begin{gathered}
p^{-1} \mathrm{~L}_{\mathrm{d} 1}[I(t)]=\mathrm{L}_{\mathrm{od}}[I(t)]=\mathrm{L}_{\mathrm{od}}[t] \mathrm{L}_{\mathrm{od}}[u(t)] \\
=p^{-2} \mathrm{~L}_{\mathrm{od}}[u(t)]=p^{-3} \mathrm{~L}_{\mathrm{d} 1}[u(t)]=p^{-3} u^{\prime}
\end{gathered}
$$

where $\mathrm{L}_{\mathrm{od}}[t]=p^{-2}$ is used. Therefore we have

$$
\mathrm{L}_{\mathrm{d} 1}[I(t)]=p^{-2} u^{\prime}
$$

or

$$
I(t)=\mathrm{L}_{\mathrm{d} 1}^{-1}\left(p^{-2} u^{\prime}\right)=\mathrm{L}_{\mathrm{od}}^{-1}\left(p^{-3} u^{\prime}\right)
$$

Namely, a number of calculations are reduced by inversion of $p^{-2} u^{\prime}$ to obtain directly $I(t)$, instead of inversion of $u^{\prime}$ followed by integration in eq 78. The remaining calculations are still lengthy but elementary in nature. We simply mention the theorem

We reach

$$
\mathrm{L}_{\mathrm{od}}^{-1}\left[(p+c)^{-n}\right]=t^{n-1}[(n-1) !]^{-1} \mathrm{e}^{-c t} \quad \text { with } \quad n \geq 1 \text { and } c \geq 0
$$

$$
R_{\mathrm{V}}=K c M\left\{\frac{8}{135} x^{-1} \varepsilon^{2} g_{2}+\frac{4}{135} a^{2} \varepsilon(s k)^{2} g_{3}+\cdots+\left[1-\frac{1}{9} a t(s k)^{2} g_{1}+\cdots\right]\right\}
$$




\section{K. NAGAI}

$$
\begin{aligned}
& R_{\mathrm{Vh}}=R_{\mathrm{Hv}}=K c M\left\{\frac{2}{45} x^{-1} \varepsilon^{2} g_{2}-\frac{11}{5670} a x^{-1} \varepsilon^{2}(s k)^{2} g_{4}-\frac{1}{630} a x^{-1} \varepsilon^{2}(\cos \theta)(s k)^{2} g_{5}+\cdots\right\} \\
& R_{\mathrm{Hh}}=K c M\left\{\frac{2}{45} x^{-1} \varepsilon^{2} g_{2}-\frac{2}{567} x^{-1} \varepsilon^{2}(s k)^{2} g_{6}+\cdots-\left(\frac{2}{45} a^{2} \varepsilon(s k)^{2} g_{7}+\cdots\right) \cos \theta\right. \\
& \left.+\left(\frac{2}{135} x^{-1} \varepsilon^{2} g_{2}-\frac{2}{135} a^{2} \varepsilon(s k)^{2} g_{8}+\cdots+\left[1-\frac{1}{9} a t(s k)^{2} g_{1}+\cdots\right]\right) \cos ^{2} \theta\right\} \\
& g_{1}(x)=1-\frac{3}{x}+\frac{6}{x^{2}}-\frac{6}{x^{3}}+\frac{6}{x^{3}} \mathrm{e}^{-x} \\
& g_{2}(x)=1-\frac{1}{3 x}+\frac{1}{3 x} \mathrm{e}^{-3 x} \\
& g_{3}(x, \varepsilon)=1-\frac{4}{63}(42+\varepsilon) \frac{1}{x}+\frac{1}{567}(1638+51 \varepsilon) \frac{1}{x^{2}}-\frac{1}{10}(30+\varepsilon) \frac{1}{x^{2}} \mathrm{e}^{-x}+\frac{1}{378}(42+5 \varepsilon) \frac{1}{x^{2}} \mathrm{e}^{-3 x} \\
& -\frac{1}{63} \frac{\varepsilon}{x} \mathrm{e}^{-3 x}-\frac{1}{315} \frac{\varepsilon}{x^{2}} \mathrm{e}^{-6 x} \\
& g_{4}(x)=1-\frac{97}{66} \frac{1}{x}+\frac{189}{110} \frac{1}{x} \mathrm{e}^{-x}-\frac{19}{66} \frac{1}{x} \mathrm{e}^{-3 x}+\frac{1}{11} \mathrm{e}^{-3 x}+\frac{13}{330} \frac{1}{x} \mathrm{e}^{-6 x} \\
& g_{5}(x)=1-\frac{87}{54} \frac{1}{x}+\frac{21}{10} \frac{1}{x} \mathrm{e}^{-x}-\frac{1}{2} \frac{1}{x} \mathrm{e}^{-3 x}-\frac{1}{3} \mathrm{e}^{-3 x}+\frac{1}{90} \frac{1}{x} \mathrm{e}^{-6 x} \\
& g_{6}(x)=1-\frac{23}{15} \frac{1}{x}+\frac{189}{100} \frac{1}{x} \mathrm{e}^{-x}-\frac{23}{60} \frac{1}{x} \mathrm{e}^{-3 x}-\frac{1}{10} \mathrm{e}^{-3 x}+\frac{2}{75} \frac{1}{x} \mathrm{e}^{-6 x} \\
& g_{7}(x, \varepsilon)=1-\frac{1}{21}(56-\varepsilon) \frac{1}{x}+\frac{1}{378}(1092-29 \varepsilon) \frac{1}{x^{2}}-\frac{1}{10}(30-\varepsilon) \frac{1}{x^{2}} \mathrm{e}^{-x}+\frac{1}{126}(14-3 \varepsilon) \frac{1}{x^{2}} \mathrm{e}^{-3 x} \\
& -\frac{1}{63} \frac{\varepsilon}{x} \mathrm{e}^{-3 x}+\frac{1}{1890} \frac{\varepsilon}{x^{2}} \mathrm{e}^{-6 x} \\
& g_{8}(x, \varepsilon)=1-\frac{1}{63}(168-2 \varepsilon) \frac{1}{x}+\frac{1}{378}(1092-17 \varepsilon) \frac{1}{x^{2}}-\frac{1}{20}(60-\varepsilon) \frac{1}{x^{2}} \mathrm{e}^{-x}+\frac{1}{756}(84-5 \varepsilon) \frac{1}{x^{2}} \mathrm{e}^{-3 x} \\
& +\frac{1}{126} \frac{\varepsilon}{x} \mathrm{e}^{-3 x}+\frac{1}{630} \frac{\varepsilon}{x^{2}} \mathrm{e}^{-6 x}
\end{aligned}
$$

with

$$
x=2 \lambda t=t / a \quad \text { and } \quad \varepsilon=\Delta \alpha / \bar{\alpha}
$$

The series in the square brackets in eq 82 and 84 is the isotropic-scattering term. Hermans and Ullman $^{13}$ neglected terms in $\varepsilon^{2}$ regarded as small compared with those in $\varepsilon$, i.e., assuming $|\varepsilon| \ll 1$. This assumption will not necessarily be valid; a large negative value of $\varepsilon$ is expected for polystyrene and its derivatives.

Expansion of $g$ into Taylor series in $x$ yields expressions for $R$ which are useful for somewhat flexible rods. (The case of rigid rods was treated by Horn, Benoit, and Oster. ${ }^{14}$ ) On the other hand, for long, flexible chains we obtain

$$
\begin{aligned}
& R_{\mathrm{Vv}}=K c M\left[1+\frac{8}{135} x^{-1} \varepsilon^{2}-\frac{1}{9} a t\left(1-\frac{3}{x}-\frac{4}{15} \frac{\varepsilon}{x}\right)(s k)^{2}\right] \\
& R_{\mathrm{Vh}}=R_{\mathrm{HV}}=K c M \cdot \frac{2}{45} x^{-1} \varepsilon^{2} \\
& R_{\mathrm{Hh}}=K c M\left\{\frac{2}{45} x^{-1} \varepsilon^{2}-\frac{2}{45} a^{2} \varepsilon(s k)^{2} \cos \theta+\left[1+\frac{2}{135} x^{-1} \varepsilon^{2}-\frac{1}{9} a t\left(1-\frac{3}{x}+\frac{2}{15} \frac{\varepsilon}{x}\right)(s k)^{2}\right] \cos ^{2} \theta\right\}
\end{aligned}
$$


In obtaining eq 94-96 we truncated the series at $(s k)^{2}$ and still ignored many terms higher in $t^{-1}$ than $t^{-1}$ with respect to $(s k)^{0}$ terms and than unity with respect to $(s k)^{2}$ terms. Eq 96 can be rearranged to

$$
\begin{aligned}
& {\left[\left(\frac{R_{\mathrm{Hh}}}{K c M}-\frac{2}{45} \frac{\varepsilon^{2}}{x}\right)(\cos \theta)^{-1}-\left(1+\frac{2}{135} \frac{\varepsilon^{2}}{x}\right) \cos \theta\right](s k)^{-2}} \\
& =-\frac{1}{9} a t\left(1-\frac{3}{x}+\frac{8}{15} \frac{\varepsilon}{x}\right)+\frac{2}{9} a t\left(1-\frac{3}{x}+\frac{2}{15} \frac{\varepsilon}{x}\right) \sin ^{2} \frac{\theta}{2}
\end{aligned}
$$

We can determine the parameters of the Porod-Kratky chain from experimental data of $R$ at low angles on polymer chains of moderate length by using eq 94,95 , and 97 , in the following way. First, $M x^{-1} \varepsilon^{2}$ is determined from $R_{\mathrm{Vh}}=R_{\mathrm{Hv}}$ or $R_{\mathrm{Hh}}(\pi / 2)$, the latter being $R_{\mathrm{Hh}}$ at $\theta=\pi / 2$. Second, $M$ and $x^{-1} \varepsilon^{2}$ are determined from $M x^{-1} \varepsilon^{2}$ and the intercept in the plot of $R_{\mathrm{Vv}}$ (or equivalently $R_{\mathrm{Vv}}^{-1}$ ) against $(s k)^{2}$. The slope in this plot gives

$$
a t\left(1-\frac{3}{x}-\frac{4}{15} \frac{\varepsilon}{x}\right)
$$

Plot of the left-hand side of eq 97 against $\sin ^{2}(\theta / 2)$ yields

$$
\text { at }\left(1-\frac{3}{x}+\frac{8}{15} \frac{\varepsilon}{x}\right) \quad \text { and } \quad a t\left(1-\frac{3}{x}+\frac{2}{15} \frac{\varepsilon}{x}\right)
$$

as its intercept and slope. [Note added in proof: the slope may be influenced by the neglected (sk) term, but the intercept is not. See ref 17 soon to appear.] From either pair of two relations out of the three we can obtain $a t\left(1-3 x^{-1}\right)$ and $a t \varepsilon x^{-1}$ (of course if the latter is not negligibly small compared with the former). Let us define

$$
C_{1}=\frac{\varepsilon^{2}}{x}, \quad C_{2}=a t\left(1-\frac{3}{x}\right), \quad \text { and } \quad C_{3}=\frac{a t \varepsilon}{x}
$$

If $C_{1}$ and $C_{3}$ are sufficiently accurate, being significantly different from zero, we can solve eq 98 to obtain

$$
\begin{aligned}
a t & =\frac{1}{2}\left[C_{2}+\left(C_{2}{ }^{2}+12 C_{1}^{-1} C_{3}^{3}\right)^{1 / 2}\right] \\
x & =3\left[1-(a t)^{-1} C_{2}\right]^{-1} \\
\varepsilon & =3 C_{3}\left(a t-C_{2}\right)^{-1}
\end{aligned}
$$

We can use $a t$ and $x=t / a$ to separate $a$ and $t$. We can split $\Delta \alpha$ from $\varepsilon=\Delta \alpha / \bar{\alpha}$ by using eq 49 combined with $\bar{\gamma}=\bar{\alpha} t$. Thus all the parameters can be determined.

The reciprocal intensities can equally be used to the same end:

$$
\begin{array}{r}
\frac{K c}{R_{\mathrm{Vv}}}=\frac{1}{M}\left[\frac{1}{1+\frac{8}{135} \frac{\varepsilon^{2}}{x}}+\frac{1}{9}\left(1-\frac{3}{x}-\frac{4}{15} \frac{\varepsilon}{x}\right)(s k)^{2}\right] \\
{\left[\frac{K c M}{R_{\mathrm{Hh}}}-\frac{1}{\frac{2}{45} \frac{\varepsilon^{2}}{x}+\left(1+\frac{2}{135} \frac{\varepsilon^{2}}{x}\right) \cos ^{2} \theta}\right] \begin{array}{r}
(\cos \theta)^{-1}(s k)^{-2}=\frac{1}{9} a t\left(1-\frac{3}{x}+\frac{8}{15} \frac{\varepsilon}{x}\right) \\
-\frac{2}{9} a t\left(1-\frac{3}{x}+\frac{2}{15} \frac{\varepsilon}{x}\right) \sin ^{2} \frac{\theta}{2}
\end{array}}
\end{array}
$$

If $C_{1}=C_{3}=0$ or $C_{1} \simeq 0$ and $C_{3} \simeq 0$, the relations eq $99-101$ are invalid or subject to great uncertainty. In these cases the only obtainable information is $C_{2}$ or three times the radius of gyration. It is imposible to separate $a$ and $t$ from data on one sample. (The chain-length dependence of $C_{2}$ should permit this.) Thus light scattering gives more information on chain conformations for 


\section{K. NAGAI}

Table I. $\mathbf{D}_{\varphi}$ (or $\mathbf{D}_{\psi}$ ) and the row and

\begin{tabular}{|c|c|c|c|c|c|c|c|c|c|c|c|}
\hline & $\stackrel{*}{y_{1}^{2}}$ & $y_{1} y_{2}$ & $y_{1} y_{3}$ & $\stackrel{*}{y_{1} y_{4}}$ & $y_{1} y_{5}$ & $\stackrel{*}{y_{1} y_{6}}$ & $\stackrel{*}{y_{2}{ }^{2}}$ & $y_{2} y_{3}$ & $y_{2} y_{4}$ & $y_{2} y_{5}$ & $y_{2} y_{6}$ \\
\hline 1 & $\mathrm{c}^{4}$ & $-2 c^{3} s$ & 0 & $c^{2} s^{2}$ & 0 & 0 & $4 c^{2} s^{2}$ & 0 & $-2 \mathrm{cs}^{3}$ & 0 & 0 \\
\hline 2 & $2 c^{3} s$ & $c^{2}\left(c^{2}-3 s^{2}\right)$ & 0 & $-\operatorname{cs}\left(c^{2}-s^{2}\right)$ & 0 & 0 & $-4 c s\left(c^{2}-s^{2}\right)$ & 0 & $s^{2}\left(3 c^{2}-s^{2}\right)$ & 0 & 0 \\
\hline 3 & 0 & 0 & $c^{3}$ & 0 & $-c^{2} s$ & 0 & 0 & $-2 c^{2} s$ & 0 & $2 \operatorname{cs}^{2}$ & 0 \\
\hline 4 & $2 c^{2} s^{2}$ & $2 \mathrm{cs}\left(\mathrm{c}^{2}-\mathrm{s}^{2}\right)$ & 0 & $c^{4}+s^{4}$ & 0 & 0 & $-8 c^{2} s^{2}$ & 0 & $-2 c s\left(c^{2}-s^{2}\right)$ & 0 & 0 \\
\hline 5 & 0 & 0 & $c^{2} s$ & 0 & $c^{3}$ & 0 & 0 & $-2 \mathrm{cs}^{2}$ & 0 & $-2 c^{2} s$ & 0 \\
\hline 6 & 0 & 0 & 0 & 0 & 0 & $c^{2}$ & 0 & 0 & 0 & 0 & $-2 \mathrm{cs}$ \\
\hline 7 & $c^{2} s^{2}$ & $\operatorname{cs}\left(c^{2}-s^{2}\right)$ & 0 & $-c^{2} s^{2}$ & 0 & 0 & $\left(c^{2}-s^{2}\right)^{2}$ & 0 & $-c s\left(c^{2}-s^{2}\right)$ & 0 & 0 \\
\hline 8 & 0 & 0 & $c^{2} s$ & 0 & $-\mathrm{cs}^{2}$ & 0 & 0 & $c\left(c^{2}-s^{2}\right)$ & 0 & $-s\left(c^{2}-s^{2}\right)$ & 0 \\
\hline 9 & $2 \mathrm{cs}^{3}$ & $s^{2}\left(3 c^{2}-s^{2}\right)$ & 0 & $\operatorname{cs}\left(c^{2}-s^{2}\right)$ & 0 & 0 & $4 \mathrm{cs}\left(\mathrm{c}^{2}-\mathrm{s}^{2}\right)$ & 0 & $c^{2}\left(c^{2}-3 s^{2}\right)$ & 0 & 0 \\
\hline 10 & 0 & 0 & $\mathrm{cs}^{2}$ & 0 & $c^{2} s$ & 0 & 0 & $s\left(c^{2}-s^{2}\right)$ & 0 & $c\left(c^{2}-s^{2}\right)$ & 0 \\
\hline 11 & 0 & 0 & 0 & 0 & 0 & cs & 0 & 0 & 0 & 0 & $\mathrm{c}^{2}-\mathrm{s}^{2}$ \\
\hline 12 & 0 & 0 & 0 & 0 & 0 & 0 & 0 & 0 & 0 & 0 & 0 \\
\hline 13 & 0 & 0 & $\mathrm{cs}^{2}$ & 0 & $-s^{3}$ & 0 & 0 & $2 c^{2} s$ & 0 & $-2 \mathrm{cs}^{2}$ & 0 \\
\hline 14 & 0 & 0 & 0 & 0 & 0 & 0 & 0 & 0 & 0 & 0 & 0 \\
\hline 15 & 0 & 0 & 0 & 0 & 0 & 0 & 0 & 0 & 0 & 0 & 0 \\
\hline 16 & $s^{4}$ & $2 \mathrm{cs}^{3}$ & 0 & $c^{2} s^{2}$ & 0 & 0 & $4 c^{2} s^{2}$ & 0 & $2 c^{3} s$ & 0 & 0 \\
\hline 17 & 0 & 0 & $\mathrm{~s}^{3}$ & 0 & $\mathrm{cs}^{2}$ & 0 & 0 & $2 \mathrm{cs}^{2}$ & 0 & $2 c^{2} s$ & 0 \\
\hline 18 & 0 & 0 & 0 & 0 & 0 & $\mathrm{~s}^{2}$ & 0 & 0 & 0 & 0 & $2 \mathrm{cs}$ \\
\hline 19 & 0 & 0 & 0 & 0 & 0 & 0 & 0 & 0 & 0 & 0 & 0 \\
\hline 20 & 0 & 0 & 0 & 0 & 0 & 0 & 0 & 0 & 0 & 0 & 0 \\
\hline \multirow[t]{2}{*}{21} & 0 & 0 & 0 & 0 & 0 & 0 & 0 & 0 & 0 & 0 & 0 \\
\hline & 1 & 2 & 3 & 4 & 5 & 6 & 7 & 8 & 9 & 10 & 11 \\
\hline
\end{tabular}

a $\mathrm{c}$ and $\mathrm{s}$ stand for $\cos \varphi$ and $\sin \varphi$. The nonvanishing (after integration) rows and columns are indicated

Table II.

\begin{tabular}{|c|c|c|c|c|c|c|c|c|c|c|c|}
\hline & $\stackrel{*}{1}$ & 2 & 3 & 娄 & 5 & 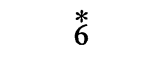 & 7 & 8 & 9 & 10 & 11 \\
\hline$* 1$ & $\mathrm{c}^{4}$ & 0 & $2 c^{3} s$ & 0 & 0 & $\mathrm{c}^{2} \mathrm{~s}^{2}$ & 0 & 0 & 0 & 0 & 0 \\
\hline 2 & 0 & $c^{3}$ & 0 & 0 & $c^{2} s$ & 0 & 0 & $2 c^{2} s$ & 0 & 0 & $\mathrm{cs}^{2}$ \\
\hline 3 & $-2 c^{3} s$ & 0 & $\mathbf{c}^{2}\left(\mathbf{c}^{2}-3 \mathrm{~s}^{2}\right)$ & 0 & 0 & $\operatorname{cs}\left(\mathrm{c}^{2}-\mathrm{s}^{2}\right)$ & 0 & 0 & 0 & 0 & 0 \\
\hline$* 4$ & 0 & 0 & 0 & $c^{2}$ & 0 & 0 & 0 & 0 & 0 & 0 & 0 \\
\hline 5 & 0 & $-c^{2} s$ & 0 & 0 & $\mathrm{c}^{3}$ & 0 & 0 & $-2 \mathrm{cs}^{2}$ & 0 & 0 & $-s^{3}$ \\
\hline * 6 & $2 c^{2} s^{2}$ & 0 & $-2 \operatorname{cs}\left(c^{2}-s^{2}\right)$ & 0 & 0 & $c^{4}+s^{4}$ & 0 & 0 & 0 & 0 & 0 \\
\hline * 7 & 0 & 0 & 0 & 0 & 0 & 0 & $c^{2}$ & 0 & 0 & cs & 0 \\
\hline 8 & 0 & $-c^{2} s$ & 0 & 0 & $-\mathrm{cs}^{2}$ & 0 & 0 & $\mathrm{c}\left(\mathrm{c}^{2}-\mathrm{s}^{2}\right)$ & 0 & 0 & $c^{2} s$ \\
\hline 9 & 0 & 0 & 0 & 0 & 0 & 0 & 0 & 0 & c & 0 & 0 \\
\hline 10 & 0 & 0 & 0 & 0 & 0 & 0 & $-2 \mathrm{cs}$ & 0 & 0 & $\mathrm{c}^{2}-\mathrm{s}^{2}$ & 0 \\
\hline 11 & 0 & $\mathrm{cs}^{2}$ & 0 & 0 & $\mathrm{~s}^{3}$ & 0 & 0 & $-2 c^{2} s$ & 0 & 0 & $c^{3}$ \\
\hline$* 12$ & $\mathbf{c}^{2} \mathbf{s}^{2}$ & 0 & $-\operatorname{cs}\left(c^{2}-s^{2}\right)$ & 0 & 0 & $-c^{2} s^{2}$ & 0 & 0 & 0 & 0 & 0 \\
\hline 13 & 0 & 0 & 0 & $-\mathrm{cs}$ & 0 & 0 & 0 & 0 & 0 & 0 & 0 \\
\hline 14 & 0 & $\mathrm{cs}^{2}$ & 0 & 0 & $-c^{2} s$ & 0 & 0 & $-\mathrm{s}\left(\mathrm{c}^{2}-\mathrm{s}^{2}\right)$ & 0 & 0 & $-\operatorname{cs}^{2}$ \\
\hline 15 & $-2 \mathrm{cs}^{3}$ & 0 & $s^{2}\left(3 c^{2}-s^{2}\right)$ & 0 & 0 & $-\operatorname{cs}\left(c^{2}-s^{2}\right)$ & 0 & 0 & 0 & 0 & 0 \\
\hline *16 & 0 & 0 & 0 & 0 & 0 & 0 & 0 & 0 & 0 & 0 & 0 \\
\hline 17 & 0 & 0 & 0 & 0 & 0 & 0 & 0 & 0 & $-\mathrm{s}$ & 0 & 0 \\
\hline *18 & 0 & 0 & 0 & $\mathrm{~s}^{2}$ & 0 & 0 & 0 & 0 & 0 & 0 & 0 \\
\hline *19 & 0 & 0 & 0 & 0 & 0 & 0 & $\mathrm{~s}^{2}$ & 0 & 0 & $-\mathrm{cs}$ & 0 \\
\hline 20 & 0 & $-s^{3}$ & 0 & 0 & $\mathrm{cs}^{2}$ & 0 & 0 & $2 \mathrm{cs}^{2}$ & 0 & 0 & $-c^{2} s$ \\
\hline$* 21$ & $s^{4}$ & 0 & $-2 \mathrm{cs}^{3}$ & 0 & 0 & $c^{2} s^{2}$ & 0 & 0 & 0 & 0 & 0 \\
\hline
\end{tabular}

a $\mathrm{c}$ and $\mathrm{s}$ stand for $\cos \theta^{\prime}$ and $\sin \theta^{\prime}$. The nonvanishing rows and columns are indicated by asterisk. 
Light Scattering by an Isotropic System Composed of Anisotropic Units

column vectors in eq $20 .^{a}$

\begin{tabular}{|c|c|c|c|c|c|c|c|c|c|c|}
\hline$y_{3}^{*}$ & $y_{3} y_{4}$ & $y_{3} y_{5}$ & $y_{3} y_{6}$ & $y_{4}^{*}$ & $y_{4} y_{5}$ & $y_{4}^{*} y_{6}$ & $y_{5}^{*}$ & $y_{5} y_{6}$ & $y_{6}^{*}$ & \\
\hline 0 & 0 & 0 & 0 & $\mathrm{~s}^{4}$ & 0 & 0 & 0 & 0 & 0 & $x_{1} x_{1}^{\prime}$ \\
\hline 0 & 0 & 0 & 0 & $-2 \mathrm{cs}^{3}$ & 0 & 0 & 0 & 0 & 0 & $x_{2} x_{1}^{\prime}+x_{1} x_{2}^{\prime}$ \\
\hline 0 & $\mathrm{cs}^{2}$ & 0 & 0 & 0 & $-s^{3}$ & 0 & 0 & 0 & 0 & $x_{3} x_{1}^{\prime}+x_{1} x_{3}^{\prime}$ \\
\hline 0 & 0 & 0 & 0 & $2 c^{2} s^{2}$ & 0 & 0 & 0 & 0 & 0 & $x_{4} x_{1}^{\prime}+x_{1} x_{4}^{\prime}$ \\
\hline 0 & $\mathrm{~s}^{3}$ & 0 & 0 & 0 & $\mathrm{cs}^{2}$ & 0 & 0 & 0 & 0 & $x_{5} x_{1}^{\prime}+x_{1} x_{5}^{\prime}$ \\
\hline 0 & 0 & 0 & 0 & 0 & 0 & $\mathrm{~s}^{2}$ & 0 & 0 & 0 & $x_{6} x_{1}^{\prime}+x_{1} x_{6}^{\prime}{ }^{*}$ \\
\hline 0 & 0 & 0 & 0 & $\mathrm{c}^{2} \mathrm{~s}^{2}$ & 0 & 0 & 0 & 0 & 0 & $x_{2} x_{2}^{\prime}$ \\
\hline 0 & $-c^{2} s$ & 0 & 0 & 0 & $\mathrm{cs}^{2}$ & 0 & 0 & 0 & 0 & $x_{3} x_{2}^{\prime}+x_{2} x_{3}^{\prime}$ \\
\hline 0 & 0 & 0 & 0 & $-2 c^{3} s$ & 0 & 0 & 0 & 0 & 0 & $x_{4} x_{2}{ }^{\prime}+x_{2} x_{4}^{\prime}$ \\
\hline 0 & $-\mathrm{cs}^{2}$ & 0 & 0 & 0 & $-c^{2} s$ & 0 & 0 & 0 & 0 & $x_{5} x_{2}^{\prime}+x_{2} x_{5}^{\prime}$ \\
\hline 0 & 0 & 0 & 0 & 0 & 0 & $-\mathrm{cs}$ & 0 & 0 & 0 & $x_{6} x_{2}{ }^{\prime}+x_{2} x_{6}^{\prime}$ \\
\hline $\mathrm{c}^{2}$ & 0 & $-\mathrm{cs}$ & 0 & 0 & 0 & 0 & $\mathrm{~s}^{2}$ & 0 & 0 & $x_{3} x_{3}{ }^{\prime}$ \\
\hline 0 & $\mathrm{c}^{3}$ & 0 & 0 & 0 & $-c^{2} s$ & 0 & 0 & 0 & 0 & $x_{4} x_{3}^{\prime}+x_{3} x_{4}^{\prime}$ \\
\hline $2 \mathrm{cs}$ & 0 & $c^{2}-s^{2}$ & 0 & 0 & 0 & 0 & $-2 \mathrm{cs}$ & 0 & 0 & $x_{5} x_{3}{ }^{\prime}+x_{3} x_{5}^{\prime}$ \\
\hline 0 & 0 & 0 & c & 0 & 0 & 0 & 0 & $-s$ & 0 & $x_{6} x_{3}{ }^{\prime}+x_{3} x_{6}^{\prime}$ \\
\hline 0 & 0 & 0 & 0 & $\mathrm{c}^{4}$ & 0 & 0 & 0 & 0 & 0 & $x_{4} x_{4}^{\prime}$ \\
\hline 0 & $c^{2} s$ & 0 & 0 & 0 & $\mathrm{c}^{3}$ & 0 & 0 & 0 & 0 & $x_{5} x_{4}^{\prime}+x_{4} x_{5}^{\prime}$ \\
\hline 0 & 0 & 0 & 0 & 0 & 0 & $\mathrm{c}^{2}$ & 0 & 0 & 0 & $x_{6} x_{4}^{\prime}+x_{4} x_{6}^{\prime}{ }^{*}$ \\
\hline $\mathrm{s}^{2}$ & 0 & cs & 0 & 0 & 0 & 0 & $\mathrm{c}^{2}$ & 0 & 0 & $x_{5} x_{5^{\prime}}$ \\
\hline 0 & 0 & 0 & $\mathrm{~s}$ & 0 & 0 & 0 & 0 & c & 0 & $x_{6} x_{5}{ }^{\prime}+x_{5} x_{6}^{\prime}$ \\
\hline 0 & 0 & 0 & 0 & 0 & 0 & 0 & 0 & 0 & 1 & $x_{6} x_{6}^{\prime}$ \\
\hline 12 & 13 & 14 & 15 & 16 & 17 & 18 & 19 & 20 & 21 & \\
\hline
\end{tabular}

by asterisk.

$\mathbf{D}_{\theta^{\prime}}$ in eq $20 .^{\text {a }}$

\begin{tabular}{cccccccccc}
\hline 12 & 13 & 14 & 15 & 16 & 17 & 18 & 19 & 20 & $21^{*}$ \\
\hline $4 \mathrm{c}^{2} \mathrm{~s}^{2}$ & 0 & 0 & $2 \mathrm{cs}^{3}$ & 0 & 0 & 0 & 0 & 0 & $\mathrm{~s}^{4}$ \\
0 & 0 & $2 \mathrm{cs}^{2}$ & 0 & 0 & 0 & 0 & 0 & $\mathrm{~s}^{3}$ & 0 \\
$4 \mathrm{cs}\left(\mathrm{c}^{2}-\mathrm{s}^{2}\right)$ & 0 & 0 & $\mathrm{~s}^{2}\left(3 \mathrm{c}^{2}-\mathrm{s}^{2}\right)$ & 0 & 0 & 0 & 0 & 0 & $2 \mathrm{cs}^{3}$ \\
0 & $2 \mathrm{cs}$ & 0 & 0 & 0 & 0 & $\mathrm{~s}^{2}$ & 0 & 0 & 0 \\
0 & 0 & $2 \mathrm{c}^{2} \mathrm{~s}$ & 0 & 0 & 0 & 0 & 0 & $\mathrm{cs}^{2}$ & 0 \\
$-8 \mathrm{c}^{2} \mathrm{~s}^{2}$ & 0 & 0 & $2 \mathrm{cs}\left(\mathrm{c}^{2}-\mathrm{s}^{2}\right)$ & 0 & 0 & 0 & 0 & 0 & $2 \mathrm{c}^{2} \mathrm{~s}^{2}$ \\
0 & 0 & 0 & 0 & 0 & 0 & 0 & $\mathrm{~s}^{2}$ & 0 & 0 \\
0 & 0 & $\mathrm{~s}\left(\mathrm{c}^{2}-\mathrm{s}^{2}\right)$ & 0 & 0 & 0 & 0 & 0 & $\mathrm{cs}^{2}$ & 0 \\
0 & 0 & 0 & 0 & 0 & $\mathrm{~s}$ & 0 & 0 & 0 & 0 \\
0 & 0 & 0 & 0 & 0 & 0 & 0 & $2 \mathrm{cs}$ & 0 & 0 \\
0 & 0 & $-2 \mathrm{cs}^{2}$ & 0 & 0 & 0 & 0 & 0 & $\mathrm{c}^{2} \mathrm{~s}$ & 0 \\
$\left(\mathrm{c}^{2}-\mathrm{s}^{2}\right)^{2}$ & 0 & 0 & $\mathrm{cs}\left(\mathrm{c}^{2}-\mathrm{s}^{2}\right)$ & 0 & 0 & 0 & 0 & 0 & $\mathrm{c}^{2} \mathrm{~s}^{2}$ \\
0 & $\mathrm{c}^{2}-\mathrm{s}^{2}$ & 0 & 0 & 0 & 0 & $\mathrm{cs}$ & 0 & 0 & 0 \\
0 & 0 & $\mathrm{c}\left(\mathrm{c}^{2}-\mathrm{s}^{2}\right)$ & 0 & 0 & 0 & 0 & 0 & $\mathrm{c}^{2} \mathrm{~s}$ & 0 \\
$-4 \mathrm{cs}\left(\mathrm{c}^{2}-\mathrm{s}^{2}\right)$ & 0 & 0 & $\mathrm{c}^{2}\left(\mathrm{c}^{2}-3 \mathrm{~s}^{2}\right)$ & 0 & 0 & 0 & 0 & 0 & $2 \mathrm{c}^{3} \mathrm{~s}$ \\
0 & 0 & 0 & 0 & 1 & 0 & 0 & 0 & 0 & 0 \\
0 & 0 & 0 & 0 & 0 & $\mathrm{c}$ & 0 & 0 & 0 & 0 \\
0 & $-2 \mathrm{cs}$ & 0 & 0 & 0 & 0 & $\mathrm{c}^{2}$ & 0 & 0 & 0 \\
0 & 0 & 0 & 0 & 0 & 0 & 0 & $\mathrm{c}^{2}$ & 0 & 0 \\
0 & 0 & $-2 \mathrm{c}^{2} \mathrm{~s}$ & 0 & 0 & 0 & 0 & 0 & $\mathrm{c}^{3}$ & 0 \\
$4 \mathrm{c}^{2} \mathrm{~s}^{2}$ & 0 & 0 & $-2 \mathrm{c}^{3} \mathrm{~s}$ & 0 & 0 & 0 & 0 & 0 & $\mathrm{c}^{4}$ \\
\hline
\end{tabular}




\section{K. NAGAI}

a short (so short that $R_{\nabla \mathrm{b}}$ takes an accurate, nonzero value) chain composed of anisotropic units than for a finite chain of isotropic units and for an infinite chain of anisotropic units. It is to be noted, however, that for very short chains eq 94-96 and the foregoing arguments cease to be valid because neglected higher order terms in $t^{-1}$ become significant.

\section{DISCUSSION}

We first return to the case of linear polymer chains of completely general type. We are particularly interested in how the procedure for analyzing experimental data, described in the preceding section, is to be modified when we consider such general chains instead of the Porod-Kratky chain. To this end we assume that chain-length dependences of $f$ for general chains are identical with those for PorodKratky chain.

Comparison of eq $52-54$ with eq $82-84$ indicates immediately

$$
f_{2} \sim n ; f_{4}, f_{5}, f_{6} \sim n ; \text { and } f_{3}, f_{7}, f_{8} \sim n^{2}
$$

It follows therefore that

$$
\begin{aligned}
& \sum_{i<j}\left\langle\left[r^{2} \operatorname{Tr} \hat{\gamma} \hat{\gamma}^{\prime}\right]_{i j}\right\rangle, \quad \sum_{i<j}\left\langle\left[\mathbf{r}^{T} \hat{\gamma} \hat{\gamma}^{\prime} \mathbf{r}\right]_{i j}\right\rangle \sim n \\
& \sum_{i<j}\left\langle\left[\bar{\gamma}\left(\mathbf{r}^{T} \hat{\gamma}^{\prime} \mathbf{r}\right)+\bar{\gamma}^{\prime}\left(\mathbf{r}^{T} \hat{\gamma} \mathbf{r}\right)\right]_{i j}\right\rangle \sim n^{2}
\end{aligned}
$$

The first relation of eq 102 was confirmed with realistic chain models., ${ }^{9,15,16}$ If only the leading terms in $f$ are retained, eq $52-54$ can be cast into eq 94-96 with the following substitutions made:

$$
\begin{aligned}
& \frac{\varepsilon^{2}}{x} \rightarrow C_{1}{ }^{\prime}=\frac{9}{4 n} \lim _{n \rightarrow \infty} n \bar{\gamma}^{-2}\left\langle\operatorname{Tr} \hat{\gamma}^{2}\right\rangle \\
& \frac{a t \varepsilon}{x} \rightarrow C_{3}{ }^{\prime}=\frac{9}{4} \lim _{n \rightarrow \infty} \bar{\gamma}^{-2} \sum_{i<j}\left\langle\left[\bar{\gamma}\left(\mathbf{r}^{T} \hat{\gamma}^{\prime} \mathbf{r}\right)+\bar{\gamma}^{\prime}\left(\mathbf{r}^{T} \hat{\gamma} \mathbf{r}\right)\right]_{i j}\right\rangle
\end{aligned}
$$$$
\text { at }\left(1-\frac{3}{x}\right) \rightarrow C_{2}{ }^{\prime}=3\left(G_{1} n-G_{0}\right)
$$

with

$$
\bar{\gamma}^{-2} \sum_{i<j}\left\langle\left[\bar{r} \bar{\gamma}^{\prime} r^{2}\right]_{i j}\right\rangle=G_{1} n-G_{0}+\mathrm{O}\left(n^{-1}\right)
$$

For a polymer chain composed of identical units the left-hand side of eq 108 reduces to $n^{-2} \sum_{i<j}\left\langle r_{i j}^{2}\right\rangle$ the radius of gyration. $C_{2}{ }^{\prime}$ is three times the radius of gyration truncated at the term of order unity. $C_{1}{ }^{\prime}, C_{2}{ }^{\prime}$, and $C_{3}{ }^{\prime}$ can be determined experimentally, and also are amenable to rigorous calculation for realistic chain models.

We proceed to compare the results for the Porod-Kratky chain or for more general chains with those for the random chain. To this end we quote results of Utiyama and Kurata's theory for the random chain. ${ }^{2}$ They obtained

$$
\begin{aligned}
& R_{\mathrm{Vv}}=K c M\left[4 \delta-2 A_{2} M Q(\theta) c+P(\theta)\right] \\
& R_{\mathrm{Vh}}=R_{\mathrm{Hv}}=3 K c M \delta \\
& R_{\mathrm{Hh}}=K c M\left\{3 \delta+\left[\delta-2 A_{2} M Q(\theta) c+P(\theta)\right] \cos ^{2} \theta\right\}
\end{aligned}
$$

For infinitely dilute solutions where interchain interactions are negligible, eq 109 and 111 simplify to

$$
\begin{aligned}
& R_{\mathrm{Vv}}=K c M[4 \delta+P(\theta)] \\
& R_{\mathrm{Hh}}=K c M\left\{3 \delta+[\delta+P(\theta)] \cos ^{2} \theta\right\}
\end{aligned}
$$

$\delta$ is given by

$$
\begin{aligned}
\delta & =\frac{B^{2}}{6 n A^{2}} \\
A & =\frac{1}{3}\left(\alpha_{1}+\alpha_{2}+\alpha_{3}\right) \\
B^{2} & =\frac{1}{15}\left[\left(\alpha_{1}-\alpha_{2}\right)^{2}+\left(\alpha_{2}-\alpha_{3}\right)^{2}+\left(\alpha_{3}-\alpha_{1}\right)^{2}\right]
\end{aligned}
$$

where $n$ is the number of random links in the random chain and $\alpha_{1}, \alpha_{2}$, and $\alpha_{3}$ are the three principal polarizabilities of each random link. $A_{2}$ is the second virial coefficient, $Q(\theta)$ is the interchain correlation function, and $P(\theta)$ is the well-known scattering function, which is expressed

$$
P(\theta)=1-\frac{1}{3}\left\langle S^{2}\right\rangle(s k)^{2}+\cdots
$$

where $\left\langle S^{2}\right\rangle$ is the mean-square radius of gyration.

From comparison of eq 112, 110, and 113, with eq 52-54 and 82-84, we find the following correspondences exist:

$$
\begin{aligned}
\delta \leftrightarrow & \frac{2}{135} \frac{\varepsilon^{2} g_{2}}{x} \leftrightarrow \frac{1}{4} \bar{\gamma}^{-2} f_{2}=\frac{1}{30} \bar{\gamma}^{-2}\left\langle\operatorname{Tr} \hat{\gamma}^{2}\right\rangle \\
P(\theta) & \leftrightarrow\left[1-\frac{1}{9} \operatorname{atg}_{1}(s k)^{2}+\cdots\right] \\
& \leftrightarrow \bar{\gamma}^{-2} \sum_{i, j}\left\langle\left[\bar{\gamma} \bar{\gamma}^{\prime} F_{0}\right]_{i j}\right\rangle
\end{aligned}
$$


Light Scattering by an Isotropic System Composed of Anisotropic Units

Some differences are also apparent. The terms involving $f_{3}, f_{7}, f_{8}$ and $f_{4}, f_{5}, f_{6}$ for general chains do not have their counterparts for the random chain. The $f_{4}, f_{5}$, and $f_{6}$ terms are usually negligible, being smaller than the $f_{3}, f_{7}$, and $f_{8}$ terms by a factor of $n^{-1}$ or $t^{-1}$, while the latter terms are not necessarily negligible.

Utiyama and Kurata ${ }^{2}$ suggested a method for deducing the mean-square radius of gyration, which is free from the influence of anisotropic scattering. From eq 112, 113, and 117 we find

$$
\begin{aligned}
R_{\mathrm{VV}}(\theta)-\frac{4}{3} R_{\mathrm{Hh}}(\pi / 2)=K c M P(\theta) & \\
=K c M[ & {\left[1-\frac{1}{3}\left\langle S^{2}\right\rangle(s k)^{2}+\cdots\right], } \\
& \text { for the random chain }
\end{aligned}
$$

Hence plot of the left-hand side (or equivalently its reciprocal) against $(s k)^{2}$ would yield $\left\langle S^{2}\right\rangle$ as its slope. On the other hand we have from eq 94 and 96

$$
\begin{array}{r}
R_{\mathrm{VV}}(\theta)-\frac{4}{3} R_{\mathrm{Hh}}(\pi / 2)=K c M\left[1-\frac{1}{9} a t\right. \\
\left.\times\left(1-\frac{3}{x}-\frac{4}{15} \frac{\varepsilon}{x}\right)(s k)^{2}+\cdots\right], \\
\quad \text { for the Porod-Kratky chain }
\end{array}
$$

By the suggested ${ }^{2}$ plot, $\left\langle S^{2}\right\rangle$ for the PorodKratky chain is overestimated for $\varepsilon<0$. The situation is similar for more general chains.

Apart from this difference the present work confirms many important aspects of Utiyama and Kurata's theory. ${ }^{2}$ When anisotropic scattering is non-negligible compared with isotropic scattering, $M$ and $\left\langle S^{2}\right\rangle$ (and possibly $A_{2}$ ) cannot be estimated correctly by the usual plots, $\lim _{\theta \rightarrow 0}$ $K c / R_{\mathrm{V}_{\mathrm{V}}}$ against $c$ and $\lim _{c \rightarrow 0} K c / R_{\mathrm{V}_{\mathrm{V}}}$ against $(\mathrm{s} k)^{2}$, and the similar plots for $K c\left(1+\cos ^{2} \theta\right) / 2 R_{\mathrm{Uu}}$, where $R_{\mathrm{Uu}}$ is $R$ for unpolarized, incident and scattered beams, i.e., $R_{\mathrm{Uu}}=\frac{1}{2}\left(R_{\mathrm{Vv}}+2 R_{\mathrm{Vh}}+R_{\mathrm{Hh}}\right)$. The correction for the anisotropic-scattering effect by a Cabannes' factor, which is valid for small molecules, is not valid any more for polymer chains. The optical anisotropy $(3 / 2)\left\langle\operatorname{Tr} \hat{\gamma}^{2}\right\rangle$ cannot be obtained from the depolarization ratios at $\theta=\pi / 2$ for polymer chains because of the influence of the intrachain interference of light, i.e., the presence of the $(s k)^{2}$ and higher terms. In the present treatment we ignored the effect of interchain interactions both optical and thermodynamic. We can take these into account formally (and approximately) in light of Utiyama and Kurata's theory. ${ }^{2}$ It seems sufficient to add the term $-2 \bar{\gamma}^{2} A_{2} M Q(\theta)$ just before the isotropic-scattering term in eq 52 and 54 , and similarly in the case of the Porod-Kratky chain.

\section{GLOSSARY OF PRINCIPAL SYMBOLS}

(Symbols referring to the Porod-Kratky chain are grouped at the end)

$A, \quad=\frac{1}{3}\left(\alpha_{1}+\alpha_{2}+\alpha_{3}\right)$, mean polarizability of the random link.

$A_{2}$, second virial coefficient.

$\mathbf{A}=\mathbf{A}_{\varphi} \mathbf{A}_{\theta}, \mathbf{A}_{\psi}$, transformation matrix correlating $X^{\prime} Y^{\prime} Z^{\prime}$ with $X Y Z$ (eq 9).

$B^{2}, \quad$ quantity related to the optical anisotropy of the random link (eq 116).

$\mathbf{B}_{\varphi}, \mathbf{B}_{\psi}$, and $\mathbf{B}_{\theta^{\prime}}$, eq 15 and 16 .

c, concentration in $\mathrm{g} / \mathrm{cc}$ of polymer.

$C_{1}{ }^{\prime}, C_{2}{ }^{\prime}$, and $C_{3}{ }^{\prime}$, eq 105-107.

$\mathbf{D}_{\varphi}, \mathbf{D}_{\psi}$, and $\mathbf{D}_{\theta^{\prime}}$, eq 20 and Tables I and II.

$F_{0}, F_{1}$, and $F_{2}$, eq $29-31$.

$f_{2}, f_{3}, \cdots, f_{8}$, eq $55-61$.

$I, \quad$ intensity of scattered light with an obvious factor omitted (eq 1).

$I_{\mathrm{HV}}$, etc., $I$ of horizontally polarized scattered light for vertically polarized incident light, etc. (eq 37-39).

$I_{\mathrm{HV}}$ (iso), $I_{\mathrm{HV}}$ (aniso), etc., isotropic and anisotropic parts of $I_{\mathrm{Hv}}$, etc., (eq $40-45$ ).

$I^{\prime}$ and $I^{\prime \prime}$, eq $3-5$.

$K, \quad$ eq 51 .

$k, \quad=2 \pi / \lambda$ with $\lambda$ the wavelength of light in the scattering medium.

$M$, molecular weight of polymer.

$n$, number of units in the scattering system or number of links in the random chain.

$P(\theta), \quad$ particle scattering function (eq 117.)

$Q(\theta)$, interchain correlation function.

$\mathbf{Q}_{0}, \mathbf{Q}_{1}$, and $\mathbf{Q}_{2}$, eq 25-27.

$R$, reduced intensity or the Rayleigh ratio (eq 51).

$R_{\mathrm{Hv}}$, etc., $R$ of horizontally polarized scattered light for vertically polarized incident light (eq 52-54).

$\mathbf{r}_{i j}$ and $r_{i j}$, distance vector from unit $i$ to unit $j$ and its magnitude. 
$\mathbf{r}$ and $r$, abbreviation of $\mathbf{r}_{i j}$ and $r_{i j}$.

$\left\langle S^{2}\right\rangle$, mean-square radius of gyration.

$\mathbf{s}, \quad=\mathbf{s}_{\mathrm{i}}-\mathbf{s}_{\mathrm{s}}$ with $\mathbf{s}_{\mathrm{i}}$ and $\mathbf{s}_{\mathrm{s}}$ the unit vectors along the incident and scattered lights (Figure 1).

$s, \quad$ absolute magnitude of $\mathbf{s}$, i.e., $2 \sin (\theta / 2)$.

U, eq 24 .

$\mathbf{U}^{\prime}, \quad$ eq 33 .

$\mathbf{V}, \quad$ eq 23.

$\mathbf{V}_{\mathrm{HV}}$, etc., $\mathbf{V}$ of horizontally polarized scattered light for vertically polarized incident light (eq 34-36).

$\mathbf{W}_{k}$, eq 47.

$x y z$, laboratory coordinate system (Figure 1).

$X Y Z$, laboratory coordinate system dependent on $\theta$ (Figure 1).

$X^{\prime} Y^{\prime} Z^{\prime}$, rotating coordinate system fixed to the scattering system.

$\mathbf{x}=\left(x_{1} \cdots x_{6}\right)^{T}$, eq 14 .

$\mathbf{x}^{\prime}=\left(x_{1}{ }^{\prime} \cdots x_{6}{ }^{\prime}\right)^{T}$, eq 18 .

$\mathbf{y}=\left(y_{1} \cdots y_{6}\right)$, eq 13 .

$\alpha_{1}, \alpha_{2}$, and $\alpha_{3}$, three principal polarizabilities of the random link.

$\boldsymbol{r}$, polarizability tensor of the total scattering system.

$\boldsymbol{\gamma}_{i}, \quad$ polarizability tensor of unit $\boldsymbol{i}$.

$\gamma_{i k},(k=1,2,3)$, three principal values of $\gamma_{i}$.

$\gamma$ and $\gamma^{\prime}$, abbreviations of $\gamma_{i k}$ and $\gamma_{j l}$.

$\bar{\gamma}$ and $\bar{\gamma}_{i}$, mean (excess) polarizabilities of the scattering system and unit $i$ (eq 48 and and below eq 61).

$\hat{\gamma}$ and $\hat{\gamma}_{i}$, traceless parts of $\boldsymbol{\gamma}$ and $\boldsymbol{\gamma}_{i}$ (below eq 61 ).

$\delta, \quad$ eq 114.

$\theta, \quad$ scattering angle (Figure 1).

$\theta^{\prime} \varphi \psi$, Eulerian angles correlating $X^{\prime} Y^{\prime} Z^{\prime}$ with $X Y Z$ (eq 9).

$\boldsymbol{\mu}_{i k},(k=1,2,3)$, unit vectors along the principal axes of $\boldsymbol{\gamma}_{i}$.

$\boldsymbol{\mu}$ and $\boldsymbol{\mu}^{\prime}$, abbreviations of $\boldsymbol{\mu}_{i k}$ and $\boldsymbol{\mu}_{j l}$.

$\boldsymbol{\mu}=\left(\mu_{1} \mu_{2} \mu_{3}\right)^{T}$ and $\boldsymbol{\mu}^{\prime}=\left(\mu_{1}^{\prime} \mu_{2}^{\prime} \mu_{3}^{\prime}\right)^{T}$, expressions of $\mu$ and $\mu^{\prime}$ in the $X^{\prime} Y^{\prime} Z^{\prime}$ system (eq 8).

$\nu$ and $\nu^{\prime}$, unit vectors along the electric vectors of scattered and incident lights respectively.

$\boldsymbol{\nu}=\left(\nu_{1} \nu_{2} \nu_{3}\right)^{T}$ and $\nu^{\prime}=\left(\nu_{1}^{\prime} \nu_{2}^{\prime} \nu_{3}^{\prime}\right)^{T}$, expressions of $\nu$ and $\nu^{\prime}$ in the $X Y Z$ system (eq 7).

$\nu_{\mathrm{V}}$ and $\nu_{\mathrm{H}}, \boldsymbol{\nu}$ for vertically and horizontally polarized scattered lights (eq 7). $\boldsymbol{\nu}_{\mathrm{v}}{ }^{\prime}$ and $\boldsymbol{\nu}_{\mathrm{h}}{ }^{\prime}, \quad \boldsymbol{\nu}^{\prime}$ for vertically and horizontally polarized incident lights (eq 7).

$\mathbf{a}^{T}, \quad$ transpose of $\mathbf{a}$.

$\mathbf{s} \cdot \mathbf{r} \equiv \mathbf{s}^{T} \mathbf{r}$, scalar product of two vectors.

$\operatorname{Tr} \gamma$, trace of a tensor, i.e., $\operatorname{Tr} \boldsymbol{\gamma}=\gamma_{11}+\gamma_{22}+\gamma_{33}$.

$\mathbf{a} \times \mathbf{b}$, direct product of two scalars, vectors, or matrices (footnote on $\mathrm{p} \mathrm{69)}$ ).

\langle\rangle , external and internal average, or internal average.

\langle\rangle$_{\text {ext }}$, external average.

\langle\rangle$_{\text {int }}$, internal average.

$\left[\begin{array}{cc}\mathrm{c}^{2} & -\mathrm{cs} \\ \mathrm{cs} & \mathrm{c}^{2}-\mathrm{s}^{2}\end{array}\right]_{\varphi} \equiv\left[\begin{array}{cc}\cos ^{2} \varphi & -\cos \varphi \sin \varphi \\ \cos \varphi \sin \varphi & \cos ^{2} \varphi-\sin ^{2} \varphi\end{array}\right]$

$\sum_{i, j}\left\langle\left[\left(F_{0}+6 F_{1}-15 F_{2}\right) r^{-2}\left(\mathbf{r}^{T} r \gamma^{\prime} \mathbf{r}\right)\right]_{i j}\right\rangle$

$\equiv \sum_{i, j}\left\langle\left[F_{0}\left(k s r_{i j}\right)+6 F_{1}\left(k s r_{i j}\right)-15 F_{2}\left(k s r_{i j}\right)\right]\right.$

$$
\left.\times \boldsymbol{r}_{i j}^{-2}\left(\mathbf{r}_{i j}{ }^{T} \boldsymbol{\gamma}_{i} \boldsymbol{\gamma}_{j} \mathbf{r}_{i j}\right)\right\rangle
$$

Porod-Kratky Chain

$a$, persistent length.

$C_{1}, C_{2}$, and $C_{3}$, eq 98 .

$f=f\left(\mathbf{r}, \mu_{t}, t\right)$, distribution function of $\mathbf{r}$ and $\boldsymbol{\mu}_{t}$.

$f^{\prime}=f^{\prime}\left(\mathbf{r}, \mu_{t}, p\right)$, dimensionless Laplace transform of $f\left(\mathbf{r}, \mu_{t}, t\right)$ (eq 67).

$g_{1}, \cdots, g_{8}$, eq 85-92.

$\mathrm{L}_{\mathrm{d} 1}$ and $\mathrm{L}_{\mathrm{d} 1}^{-1}$, dimensionless Laplace transform operator and its inverse operator (eq 67).

$\mathrm{L}_{\mathrm{od}}$ and $\mathrm{L}_{\mathrm{od}}^{-1}$, ordinary Laplace transform operator and its inverse operator (eq. 69).

$p$, Laplace transform parameter (eq 67).

$\mathbf{r}$ and $r$, end-to-end vector and its magnitude.

$t$, contour length.

$u_{k l m n}=\langle\Psi\rangle$, eq 66 .

$u_{k l m n}^{\prime}=\langle\Psi\rangle^{\prime}, \quad=\mathrm{L}_{\mathrm{d} 1}\left[u_{k l m n}\right]$.

$x, \quad=2 \lambda t=t / a$.

$\alpha_{1}$ and $\alpha_{2}$, longitudinal and transverse polarizabilities per unit length.

$\bar{\alpha}$ and $\Delta \alpha$, mean $\left[\bar{\alpha}=\frac{1}{3}\left(\alpha_{1}+2 \alpha_{2}\right)\right]$ and anisotropic $\left(\Delta \alpha=\alpha_{1}-\alpha_{2}\right)$ polarizabilities per unit length.

$\varepsilon, \quad=\Delta \alpha / \bar{\alpha}$, degree of anisotropy of polarizability per unit length.

$\theta$ and $\varphi$, polar coordinates of $\boldsymbol{\mu}_{t}$.

$\boldsymbol{\mu}_{i}$, tangent at the point that departs by length $i$ from one end along the chain contour.

$\lambda, \quad=1 /(2 a)$. 


\section{REFERENCES}

1. P. Debye, J. Appl. Phys., 15, 338 (1944); J. Phys. \& Colloid Chem., 51, 18 (1947).

2. (a) H. Utiyama and M. Kurata, Bull. Inst. Chem. Res. Kyoto Univ., 42, 128 (1964); (b) H. Utiyama, J. Phys. Chem., 69, 4138 (1965).

3. P. Debye and A. M. Bueche, J. Appl. Phys., 20, 518 (1949).

4. M. Goldstein and E. R. Michalik, J. Appl. Phys., 26, 1450 (1955).

5. R. S. Stein and P. R. Wilson, J. Appl. Phys., 33, 1914 (1962).

6. K. Nagai, J. Chem. Phys., 38, 924 (1963).

7. K. Nagai and T. Ishikawa, J. Chem. Phys., 45, 3128 (1966).

8. P. J. Flory, "Principles of Polymer Chemistry," Cornell Univ. Press, Ithaca, N.Y., 1953,
Chapter VII.

9. P. J. Flory, "Statistical Mechanics of Chain Molecules," Interscience, New York, N.Y., 19.69, Chapter IX.

10. Ref. 9, Chapter VIII.

11. H. Maeda, N. Saito, and W. H. Stockmayer, Polymer J., 2, 94 (1971).

12. G. Porod, Monatsh. Chem., 80, 251 (1949); O. Kratky and G. Porod, Rec. Trav. Chim., 68, 1106 (1949).

13. J. J. Hermans and R. Ullman, Physica, 18, 951 (1952).

14. P. Horn, H. Benoit, and G. Oster, J. Chim. Phys., 48, 530 (1951).

15. R. L. Jernigan and P. J. Flory, J. Chem. Phys., 47, 1999 (1967).

16. K. Nagai, J. Chem. Phys., 47, 4690 (1967).

17. K. Nagai, Polymer J., submitted. 